\title{
Homogenization of linearized elasticity in a two-component medium with slip displacement conditions
}

\author{
Tanja Lochner ${ }^{\mathrm{a}, *}$, Malte A. Peter ${ }^{\mathrm{a}, \mathrm{b}}$ \\ ${ }^{a}$ Institute of Mathematics, University of Augsburg, 86135 Augsburg, Germany \\ ${ }^{b}$ Augsburg Centre for Innovative Technologies, University of Augsburg, 86135 Augsburg, Germany
}

\begin{abstract}
We consider the periodic homogenization of the linearized elasticity problem with slip displacement conditions for a two-scale composite of two solids. Such interface jumps in displacement arise e.g. in contact problems with imperfect bonding. Motivated by applications in carbon-fibre-reinforced concretes, we aim to investigate the impact of the length of the carbon fibres on the effective properties, which is why we assume one of the materials to be connected, whereas the other one is either connected or disconnected. The method of periodic unfolding is applied, deriving some new compactness results in passing, to determine the macroscopic limit problems rigorously, which show significant differences for the two cases.
\end{abstract}

Keywords: periodic homogenization, linear elasticity, imperfect interface, slip displacement

\section{Introduction}

In a two-scale composite of two solids, we consider the upscaling in the context of periodic homogenization of the linear elasticity problem with slip displacement conditions in normal and tangential direction at the internal interface, and Dirichlet and Neumann boundary conditions at separate parts of the outer boundary. While one of the components is assumed globally connected, we consider two distinct cases for the other component: globally connected or disconnected.

The problem is motivated by recently proposed concretes reinforced with short carbon fibres, cf. [1], 2], 3]. Similarly to carbon-fibre-reinforced ceramics, the bonding of the fibres to the concrete matrix is rather weak, so that it cannot be modelled as a perfect bond. Hence, we apply general linear slip displacement conditions. Moreover, the carbon fibres in this new material occur as single fibres and they are rather short (of the order of $1 \mathrm{~cm}$ in length). Therefore, the question arises what connectivity should be assumed in the homogenization setting. We investigate the impact of this choice by considering the two different connectivities alluded to above as extreme cases.

Related to our choice of internal interface condition, there are several authors studying homogenization problems with jumps on the interface and focus on the disconnectedness of one of the subdomains. In [4], [5] and [6], thermal diffusion with homogeneous Dirichlet boundary conditions

\footnotetext{
*Corresponding author

Email addresses: tanja.lochner@math.uni-augsburg.de (Tanja Lochner), malte.peter@math. uni-augsburg.de (Malte A. Peter)
} 
on the outer boundary is considered. To derive the homogenized solution in the first paper, Tartar's method of oscillating test functions [7] was applied and, in the second and third, the periodic unfolding method [8] was used. Moreover, in this scalar diffusion setting, scaling of the interfacial term was considered systematically in [9] in terms of two-scale convergence [10, 11]. Nonlinear variants of these problems were considered more recently by [12], [13] and [14]. In [15], the (vector-valued) elasticity problem with prescribed interface jumps in displacements and tractions and Neumann condition on a part of the external boundary was studied. A related vector-valued problem with perfect bonding was recently considered by [16].

As mentioned above, the connectivity of the domain is known to have a significant impact. This is highlighted in [9] in terms of two-scale-convergence compactness results. We refer to [4], [5], [12] and [6] for the impact in terms of the upscaled system of equations.

Although the periodic homogenization of linearized elasticity is well-known for standard (internal and external) boundary conditions in the literature, cf. e.g. [17] and [18], difficulties arise due to the fact that the slip displacement conditions only yield Robin-type interface conditions, which complicates the a-priori estimates of the symmetric gradient. In the disconnected case, we can resolve this issue by choosing an appropriate solution space and using standard extension operators. In the connected case, we use the recently developed extension operators from [19] to handle the homogeneous Dirichlet boundary conditions at the external boundary.

The paper is organized as follows. In section 2, we state the problem for a composite with periodic microstructure, for which we prove the existence and uniqueness of the solution in the connected and disconnected case in section 3. In section 4, we derive the homogenized problems in both cases, where general compactness results are proven first, which may be of general interest independent of the particular boundary-value problem considered here. A comparison and discussion of the limit problems is given in section 5 .

\section{Statement of the problem for a composite with periodic microstructure}

We consider a composite of two materials with a periodic microstructure and distinguish two cases. In the disconnected case, one material is connected, whereas the other one is not. On the other hand, in the connected case, the composite consists of two globally connected materials. We choose a bounded connected Lipschitz-domain $\Omega \subset \mathbb{R}^{3}$, which can be represented as a union of axis-parallel cuboids and which has corner coordinates in $\mathbb{Q}^{3}$.

In order to describe the periodic microstructure, we define the reference cell $Y=[0,1]^{3} \subset \mathbb{R}^{3}$ and two subsets $Y_{0}, Y_{1} \subset Y$ such that $Y_{0}^{\circ} \cap Y_{1}^{\circ}=\emptyset, \Sigma_{Y}:=\overline{Y_{0}} \cap \overline{Y_{1}}$ Lipschitz-continuous and $Y=Y_{0} \cup \Sigma_{Y} \cup Y_{1}$. In the disconnected case, we assume that $Y_{0} \subset \subset Y$, i.e. $\partial Y_{0} \cap \partial Y=\emptyset$, and, in the connected one, we prescribe that the boundary of $Y_{0}$ resp. $Y_{1}$ on opposite faces is the same and if we extend $Y$ 1-periodically, this splits the space into two connected sets. We define the following sets:

- $\Omega_{\alpha}^{\varepsilon}:=\bigcup_{\xi \in \Lambda^{\varepsilon}} \varepsilon\left(Y_{\alpha}+\xi\right)$ is the $Y$-periodically extended domain $Y_{\alpha}$ scaled with $\varepsilon, \alpha=0,1$,

- $\Sigma^{\varepsilon}:=\bigcup_{\xi \in \Lambda^{\varepsilon}} \varepsilon\left(\Sigma_{Y}+\xi\right)$ is the $Y$-periodically extended interface $\Sigma_{Y}$ scaled with $\varepsilon$,

- $\Omega^{\varepsilon}=\Omega_{0}^{\varepsilon} \cup \Omega_{1}^{\varepsilon} \cup \Sigma^{\varepsilon}$ is the union of the scaled cells,

where $\Lambda^{\varepsilon}:=\left\{\xi \in \mathbb{Z}^{3}: \varepsilon(Y+\xi) \subset \Omega\right\}$. The idea is that $\Omega_{0}^{\varepsilon}$ represents one material and $\Omega_{1}^{\varepsilon}$ the other one. We split the external boundary into two parts $\partial \Omega=\Gamma_{1} \cup \Gamma_{2}$, where $\Gamma_{1}$ and $\Gamma_{2}$ are disjoint sets 
and $\Gamma_{1}$ has positive measure. In both cases, we only admit the scaling factor $\varepsilon$ in such a way that $\varepsilon^{-1} \Omega$ can be represented as a finite union of axis-parallel cuboids with corner coordinates in $\mathbb{Z}^{3}$. Thus, we can guarantee that the domain $\Omega$ can completely be filled up with scaled reference cells. This condition can be relaxed but we assume it in what follows to avoid well-known technicalities induced by otherwise non-matching boundaries of $\Omega$ and its $\varepsilon$-periodic approximation. Due to the choice of $\varepsilon$, there holds $\Omega^{\varepsilon}=\Omega, \Gamma_{1}=\Gamma_{1} \cap \partial \Omega^{\varepsilon}$ and $\Gamma_{2}=\Gamma_{2} \cap \partial \Omega^{\varepsilon}$. Let $n$ be the normal to $\Sigma^{\varepsilon}$ with orientation from $\Omega_{0}^{\varepsilon}$ to $\Omega_{1}^{\varepsilon}$ and $\nu$ the outward-pointing normal to $\Gamma_{2}$.

We consider the linear elasticity equation with slip displacement conditions on the interface of the materials. We let $u_{\varepsilon}: \Omega^{\varepsilon} \rightarrow \mathbb{R}^{3}$ be the displacement field. The stress tensor $\sigma^{\varepsilon}=\left(\sigma_{i j}^{\varepsilon}\right)_{1 \leq i, j \leq 3}$ is defined by

$$
\sigma_{i j}^{\varepsilon}:=\sum_{k, l=1}^{3} a_{i j k l}^{\varepsilon} e_{k l}\left(u^{\varepsilon}\right):=\sum_{k, l=1}^{3} a_{i j k l}^{\varepsilon} \frac{1}{2}\left(\partial_{k} u_{l}^{\varepsilon}+\partial_{l} u_{k}^{\varepsilon}\right),
$$

where the symmetric gradient $e\left(u^{\varepsilon}\right)=\left(e_{k l}\left(u^{\varepsilon}\right)\right)_{1<k, l<3}$ is the linear strain tensor and $A^{\varepsilon}(x)=$ $\left(a_{i j k h}^{\varepsilon}(x)\right)_{1 \leq i, j, k, h \leq 3}$ is a tensor of fourth order, which describes the properties of the materials of the solid. In order to formulate the slip displacement conditions, we introduce some additional notation:

- $u_{n}^{\varepsilon}:=u^{\varepsilon} \cdot n$ resp. $u_{\tau^{i}}^{\varepsilon}:=u^{\varepsilon} \cdot \tau^{i}, i=1,2$, the projection of the displacement field in normal resp. tangential direction of the interface,

- $\sigma_{n}^{\varepsilon}:=\left(\sigma^{\varepsilon} \cdot n\right) \cdot n$ resp. $\sigma_{\tau^{i}}^{\varepsilon}:=\left(\sigma^{\varepsilon} \cdot n\right) \cdot \tau^{i}, i=1,2$, the projection of the normal stress in normal resp. tangential direction of the interface,

- $[\varphi]_{\Sigma^{\varepsilon}}:=\left.\left(\varphi_{1}-\varphi_{0}\right)\right|_{\Sigma^{\varepsilon}}$ the jump on the interface,

where $\varphi_{\alpha}:=\left.\varphi\right|_{\Omega_{\alpha}^{\varepsilon}}$ is the restriction of $\varphi$ to $\Omega_{\alpha}^{\varepsilon}$ and $\left.\varphi_{\alpha}\right|_{\Sigma^{\varepsilon}}$ is the trace of $\varphi_{\alpha}$ (we write only $\varphi_{\alpha}$ if it is clear) for $\alpha=0,1$.

With this notation, the linear elasticity problem is given by

$$
\left\{\begin{aligned}
-\nabla \cdot \sigma^{\varepsilon} & =f^{\varepsilon} & & \text { in } \Omega_{0}^{\varepsilon} \cup \Omega_{1}^{\varepsilon}, \\
u^{\varepsilon} & =0 & & \text { on } \Gamma_{1}, \\
\sigma^{\varepsilon} \cdot \nu & =g & & \text { on } \Gamma_{2},
\end{aligned}\right.
$$

with the slip displacement condition on the interface $\Sigma^{\varepsilon}$

$$
\left\{\begin{aligned}
\varepsilon\left[u_{n}^{\varepsilon}\right]_{\Sigma^{\varepsilon}} & =\frac{1}{K_{\mathrm{N}}} \sigma_{n}^{\Sigma^{\varepsilon}} \\
\varepsilon\left[u_{\tau^{i}}^{\varepsilon}\right]_{\Sigma^{\varepsilon}} & =\frac{1}{K_{\mathrm{T}}} \sigma_{\tau^{i}}^{\Sigma^{\varepsilon}}, i=1,2 \\
{\left[\sigma_{n}^{\varepsilon}\right]_{\Sigma^{\varepsilon}} } & =0 \\
{\left[\sigma_{\tau_{i}}^{\varepsilon}\right]_{\Sigma^{\varepsilon}} } & =0, i=1,2
\end{aligned}\right.
$$

where the constants $K_{\mathrm{N}}, K_{\mathrm{T}}>0$ are the normal and tangential stiffness, respectively, and $\sigma^{\Sigma^{\varepsilon}}$ is the stress tensor of the interface. We refer to [20] for more details on the slip displacement conditions. 
For simplification, we choose $A^{\varepsilon} \in M(\alpha, \beta, Y)$ (see Definition 2.1 below) with

$$
A^{\varepsilon}(x)=\left(a_{i j k h}^{\varepsilon}(x)\right)_{1 \leq i, j, k, h \leq 3}:=\left(a_{i j k h}\left(\frac{x}{\varepsilon}\right)\right)_{1 \leq i, j, k, h \leq 3}=A\left(\frac{x}{\varepsilon}\right),
$$

where $A(\cdot)=\left(a_{i j k h}(\cdot)\right)_{1 \leq i, j, k, h \leq 3} \in M(\alpha, \beta, Y)$ and all components $a_{i j k h}$ are $Y$-periodic for all $i, j, k, h \in\{1,2,3\}$. Under additional assumptions we can assume a more general tensor $A$ dependent on both the microscopic and the macroscopic coordinate, see Remark 4.15 and Remark 4.21.

Definition 2.1. Let $\alpha, \beta \in \mathbb{R}$ with $0<\alpha<\beta$ and let $\mathcal{O}$ be an open set in $\mathbb{R}^{3}$. We denote by $M(\alpha, \beta, \mathcal{O})$ the set of all tensors $B=\left(b_{i j k h}\right)_{1 \leq i, j, k, h \leq 3}$ such that

(i) $b_{i j k h} \in L^{\infty}(\mathcal{O})$ for all $i, j, k, h \in\{1,2,3\}$

(ii) $b_{i j k h}=b_{j i k h}=b_{k h i j}$ for all $i, j, k, h \in\{1,2,3\}$

(iii) $\alpha|m|^{2} \leq B m m$ for all symmetric matrices $m$

(iv) $|B(x) m| \leq \beta|m|$ for all matrices $m$

a.e. in $\mathcal{O}$, where

$$
\left\{\begin{array}{l}
B m:=\left((B m)_{i j}\right)_{1 \leq i, j \leq 3}=\left(\left(\sum_{k, h=1}^{3} B_{i j k h} m_{k h}\right)_{i j}\right)_{1 \leq i, j \leq 3} \\
B m \tilde{m}:=\sum_{i, j, k, h=1}^{3} b_{i j k h} m_{i j} \tilde{m}_{k h}, \\
|m|:=\left(\sum_{i, j=1}^{3} m_{i j}^{2}\right)^{\frac{1}{2}}
\end{array}\right.
$$

for quadratic matrices $m=\left(m_{i j}\right)_{1 \leq i, j \leq 3}$ and $\tilde{m}=\left(\tilde{m}_{i j}\right)_{1 \leq i, j \leq 3}$.

We test problem (1) with some sufficiently smooth function $\varphi$, use integration by parts, the boundary conditions, the fact that $\sigma^{\varepsilon}$ is symmetric and the jump of the normal stress on $\Sigma^{\varepsilon}$ is zero, meaning that $\sigma_{0}^{\varepsilon}=\sigma_{1}^{\varepsilon}$ on $\Sigma^{\varepsilon}$ and thus $\sigma_{0}^{\varepsilon}, \sigma_{1}^{\varepsilon}$ coincide with $\sigma^{\Sigma^{\varepsilon}}$ on $\Sigma^{\varepsilon}$, to obtain the weak formulation

$$
\begin{aligned}
\int_{\Omega_{0}^{\varepsilon}} A^{\varepsilon} e\left(u_{0}^{\varepsilon}\right) e\left(\varphi_{0}\right) \mathrm{d} x+\int_{\Omega_{1}^{\varepsilon}} A^{\varepsilon} e\left(u_{1}^{\varepsilon}\right) e\left(\varphi_{1}\right) \mathrm{d} x \\
\quad+\varepsilon \int_{\Sigma^{\varepsilon}}\left(K_{\mathrm{N}}\left[u_{n}^{\varepsilon}\right]_{\Sigma^{\varepsilon}} n+K_{\mathrm{T}} \sum_{i=1}^{2}\left[u_{\tau^{i}}^{\varepsilon}\right]_{\Sigma^{\varepsilon}} \tau^{i}\right) \cdot\left(\varphi_{1}-\varphi_{0}\right) \mathrm{d} S(x) \\
=\int_{\Omega_{0}^{\varepsilon}} f^{\varepsilon} \cdot \varphi_{0} \mathrm{~d} x+\int_{\Omega_{1}^{\varepsilon}} f^{\varepsilon} \cdot \varphi_{1} \mathrm{~d} x+\int_{\Gamma_{2} \cap \partial \Omega_{0}^{\varepsilon}} g \cdot \varphi_{0} \mathrm{~d} S(x)+\int_{\Gamma_{2} \cap \partial \Omega_{1}^{\varepsilon}} g \cdot \varphi_{1} \mathrm{~d} S(x) .
\end{aligned}
$$

Note that we have not yet introduced the function spaces, which complete the weak formulation. This is done in the next section and it will turn out that different function spaces are required depending on the connectedness of $\Omega_{0}^{\varepsilon}$. Throughout the paper, we denote by $C$ a constant independent of $\varepsilon$ whose value may change from line to line and we write $\mathcal{D}(\Omega):=\mathcal{C}_{0}^{\infty}(\Omega)$ and $\mathcal{D}(\bar{\Omega}):=\mathcal{C}^{\infty}(\Omega)$.

\section{Existence of solutions}

Before we can apply homogenization methods to obtain the upscaled problem, we have to justify the existence, uniqueness and uniform boundedness of the solution. In subsection 3.1, we introduce the complete weak form and we prove the result for the disconnected case and in subsection 3.2 for the connected case. 


\subsection{Existence result for disconnected case}

First of all, we notice that $\Gamma_{2} \cap \partial \Omega_{0}^{\varepsilon}=\emptyset$ and $\Gamma_{2} \cap \partial \Omega_{1}^{\varepsilon}=\Gamma_{2}$ in the disconnected case, so the third term on the right-hand side in (3) disappears. Since infinitesimal rotations do not induce forces, we assume that there are no rotations, meaning that the curl, $\nabla \times \cdot$, has to be zero. Therefore, we define the solution space for the disconnected case as

$$
\mathcal{W}_{\mathrm{d}}\left(\Omega^{\varepsilon}\right)=\left\{u \in\left[L^{2}\left(\Omega^{\varepsilon}\right)\right]^{3}: u_{0} \in\left[H^{1}\left(\Omega_{0}^{\varepsilon}\right)\right]^{3}, u_{1} \in\left[H^{1}\left(\Omega_{1}^{\varepsilon}, \Gamma_{1}\right)\right]^{3}, \nabla \times u_{0}=0 \text { in } \Omega_{0}^{\varepsilon}\right\},
$$

endowed with the norm

$$
\|u\|_{\mathcal{W}_{\mathrm{d}}\left(\Omega^{\varepsilon}\right)}^{2}:=\left\|e\left(u_{0}\right)\right\|_{\left[L^{2}\left(\Omega_{0}^{\varepsilon}\right)\right]^{3 \times 3}}^{2}+\left\|e\left(u_{1}\right)\right\|_{\left[L^{2}\left(\Omega_{1}^{\varepsilon}\right)\right]^{3 \times 3}}^{2}+\varepsilon\left\|[u]_{\Sigma^{\varepsilon}}\right\|_{\left[L^{2}\left(\Sigma^{\varepsilon}\right)\right]^{3}}^{2}
$$

for all $u \in \mathcal{W}_{\mathrm{d}}\left(\Omega^{\varepsilon}\right)$, whereby $H^{1}(\mathcal{O}, \tilde{\Gamma}):=\left\{u \in H^{1}(\mathcal{O}): u=0\right.$ on $\left.\tilde{\Gamma}\right\}$ for some open set $\mathcal{O}$ with $\tilde{\Gamma} \subset \partial \mathcal{O}$. In what follows, we often use the fact that

$$
\nabla u_{0}=\frac{1}{2}\left(\nabla u_{0}+\left(\nabla u_{0}\right)^{T}\right)+\frac{1}{2}\left(\nabla u_{0}-\left(\nabla u_{0}\right)^{T}\right)=\frac{1}{2}\left(\nabla u_{0}+\left(\nabla u_{0}\right)^{T}\right)=e\left(u_{0}\right)
$$

for all $u \in \mathcal{W}_{\mathrm{d}}\left(\Omega^{\varepsilon}\right)$. Before we prove that $\mathcal{W}_{\mathrm{d}}\left(\Omega^{\varepsilon}\right)$ is a Banach space, we gather some estimates.

Lemma 3.1. For every $v \in \mathcal{W}_{\mathrm{d}}\left(\Omega^{\varepsilon}\right)$, there holds

(i) $\varepsilon\left\|v_{1}\right\|_{\left[L^{2}\left(\Sigma^{\varepsilon}\right)\right]^{3}}^{2} \leq C\left(\left\|v_{1}\right\|_{\left[L^{2}\left(\Omega_{1}^{\varepsilon}\right)\right]^{3}}^{2}+\varepsilon^{2}\left\|\nabla v_{1}\right\|_{\left[L^{2}\left(\Omega_{1}^{\varepsilon}\right)\right]^{3 \times 3}}^{2}\right)$

(ii) $\left\|v_{0}\right\|_{\left[L^{2}\left(\Omega_{0}^{\varepsilon}\right)\right]^{3}}^{2} \leq C\left(\varepsilon^{2}\left\|e\left(v_{0}\right)\right\|_{\left[L^{2}\left(\Omega_{0}^{\varepsilon}\right)\right]^{3 \times 3}}^{2}+\varepsilon\left\|v_{0}\right\|_{\left[L^{2}\left(\Sigma^{\varepsilon}\right)\right]^{3}}^{2}\right)$

for constants $C>0$ independent of $\varepsilon$.

Proof. Both estimates follow by scaling and summation from standard results for the trace operator (for (i)) and Korn's inequality from [21, Corollary 5.8] (for (ii)).

Theorem 3.2. $\left(\mathcal{W}_{\mathrm{d}}\left(\Omega^{\varepsilon}\right),\|u\|_{\mathcal{W}_{\mathrm{d}}\left(\Omega^{\varepsilon}\right)}\right)$ defines a Banach space.

Proof. Using the fact that $u_{1}=0$ on $\Gamma_{1}$ for $u \in \mathcal{W}_{\mathrm{d}}\left(\Omega^{\varepsilon}\right)$, it is easily proven that $\|\cdot\|_{\mathcal{W}_{\mathrm{d}}\left(\Omega^{\varepsilon}\right)}^{2}$ defines a norm on $\mathcal{W}_{\mathrm{d}}\left(\Omega^{\varepsilon}\right)$. Let $\left\{u^{(k)}\right\}_{k}$ be a Cauchy sequence in $\mathcal{W}_{\mathrm{d}}\left(\Omega^{\varepsilon}\right)$. With the help of [17, Theorem 4.2], we can construct a global extension operator on domains with holes, which do not intersect the boundary of the reference cell. More precisely, there exists a linear extension operator $P_{\varepsilon}:\left[H^{1}\left(\Omega_{1}^{\varepsilon}\right)\right]^{3} \rightarrow\left[H^{1}(\Omega)\right]^{3}$ such that

$$
\begin{aligned}
& \left\|P_{\varepsilon} v\right\|_{\left[H^{1}(\Omega)\right]^{3}} \leq c_{1}\|v\|_{\left[H^{1}\left(\Omega_{1}^{\varepsilon}\right)\right]^{3}}, \\
& \left\|P_{\varepsilon} v\right\|_{\left[L^{2}(\Omega)\right]^{3}}+\left\|e\left(P_{\varepsilon} v\right)\right\|_{\left[L^{2}(\Omega)\right]^{3 \times 3}} \leq c_{2}\left(\|v\|_{\left[L^{2}\left(\Omega_{1}^{\varepsilon}\right)\right]^{3}}+\|e(v)\|_{\left[L^{2}\left(\Omega_{1}^{\varepsilon}\right)\right]^{3 \times 3}}\right), \\
& \left\|e\left(P_{\varepsilon} v\right)\right\|_{\left[L^{2}(\Omega)\right]^{3 \times 3}} \leq c_{3}\|e(v)\|_{\left[L^{2}\left(\Omega_{1}^{\varepsilon}\right)\right]^{3 \times 3}}
\end{aligned}
$$

for all $v \in\left[H^{1}\left(\Omega_{1}^{\varepsilon}\right)\right]^{3}$, where the constants $c_{1}, c_{2}, c_{3}$ do not depend on $\varepsilon$. Korn's inequality for functions with zero trace on part of the boundary yields

$$
\left\|u_{1}\right\|_{\left[H^{1}\left(\Omega_{1}^{\varepsilon}\right)\right]^{3}}^{2} \leq\left\|P_{\varepsilon} u_{1}\right\|_{\left[H^{1}(\Omega)\right]^{3}}^{2} \leq C\left\|e\left(P_{\varepsilon} u_{1}\right)\right\|_{\left[L^{2}(\Omega)\right]^{3 \times 3}}^{2} \leq C\left\|e\left(u_{1}\right)\right\|_{\left[L^{2}\left(\Omega_{1}^{\varepsilon}\right)\right]^{3 \times 3}}^{2} .
$$


Using this inequality twice and Lemma 3.1 (i) and (ii), we get for all $u \in \mathcal{W}_{\mathrm{d}}\left(\Omega^{\varepsilon}\right)$

$$
\begin{aligned}
& \left\|u_{0}\right\|_{\left[H^{1}\left(\Omega_{0}^{\varepsilon}\right)\right]^{3}}^{2}+\left\|u_{1}\right\|_{\left[H^{1}\left(\Omega_{1}^{\varepsilon}\right)\right]^{3}}^{2} \\
& \left.\quad \leq C\left(\left(1+\varepsilon^{2}\right)\left\|e\left(u_{0}\right)\right\|_{\left[L^{2}\left(\Omega_{0}^{\varepsilon}\right)\right.}^{2}\right]^{3 \times 3}+\varepsilon\left\|[u]_{\Sigma^{\varepsilon}}\right\|_{\left[L^{2}\left(\Sigma^{\varepsilon}\right)\right]^{3}}^{2}+\varepsilon\left\|u_{1}\right\|_{\left[L^{2}\left(\Sigma^{\varepsilon}\right)\right]^{3}}^{2}+\left\|e\left(u_{1}\right)\right\|_{\left[L^{2}\left(\Omega_{1}^{\varepsilon}\right)\right]^{3 \times 3}}^{2}\right) \\
& \quad \leq C\|u\|_{\mathcal{W}_{\mathrm{d}}\left(\Omega^{\varepsilon}\right)}^{2} .
\end{aligned}
$$

Therefore, $\left\{u_{0}^{(k)}\right\}_{k}$ and $\left\{u_{1}^{(k)}\right\}_{k}$ are Cauchy sequences in the Banach spaces $H^{1}\left(\Omega_{0}^{\varepsilon}\right)$ resp. $H^{1}\left(\Omega_{1}^{\varepsilon}\right)$. Thus there exits $u_{0} \in H^{1}\left(\Omega_{0}^{\varepsilon}\right)$ and $u_{1} \in H^{1}\left(\Omega_{1}^{\varepsilon}\right)$ with $u_{0}^{(k)} \rightarrow u_{0}$ and $u_{1}^{(k)} \rightarrow u_{1}$ for $k \rightarrow \infty$. It remains to show that $u=\left(u_{0}, u_{1}\right) \in \mathcal{W}_{\mathrm{d}}\left(\Omega^{\varepsilon}\right)$. Due to the continuity of the trace operator, $u_{1}$ is equal to zero on $\Gamma_{2}$. Using the convergence of $\left\{u_{0}^{(k)}\right\}_{k}$ and the fact that $\nabla u_{0}^{(k)}=e\left(u_{0}^{(k)}\right)$, we get

$$
\partial_{x_{j}}\left(u_{0}\right)_{i}=\lim _{k \rightarrow \infty} \partial_{x_{j}}\left(u_{0}^{(k)}\right)_{i}=\lim _{k \rightarrow \infty} \frac{1}{2}\left(\partial_{x_{j}}\left(u_{0}^{(k)}\right)_{i}+\partial_{x_{i}}\left(u_{0}^{(k)}\right)_{j}\right)=\frac{1}{2}\left(\partial_{x_{j}}\left(u_{0}\right)_{i}+\partial_{x_{i}}\left(u_{0}\right)_{j}\right)
$$

for all $i, j \in\{1,2,3\}$, which shows that $\nabla \times u_{0}=0$.

Theorem 3.3. Let $f^{\varepsilon} \in\left[L^{2}\left(\Omega^{\varepsilon}\right)\right]^{3}, g \in\left[L^{2}\left(\Gamma_{2}\right)\right]^{3}$. Then, there exists a unique weak solution $u \in \mathcal{W}_{\mathrm{d}}\left(\Omega^{\varepsilon}\right)$ of (3) for all admissible $0<\varepsilon \leq 1$.

Proof. Our aim is to apply the Lax-Milgram Theorem. Let $\varepsilon>0$. We denote the left-hand side of (3) as a mapping $a: \mathcal{W}_{\mathrm{d}}\left(\Omega^{\varepsilon}\right) \times \mathcal{W}_{\mathrm{d}}\left(\Omega^{\varepsilon}\right) \rightarrow \mathbb{R}$

$$
\begin{aligned}
a(u, v)=\int_{\Omega_{0}^{\varepsilon}} A^{\varepsilon} e\left(u_{0}\right) e\left(v_{0}\right) \mathrm{d} x+\int_{\Omega_{1}^{\varepsilon}} A^{\varepsilon} e\left(u_{1}\right) e\left(v_{1}\right) \mathrm{d} x \\
\quad+\varepsilon \int_{\Sigma^{\varepsilon}}\left(K_{\mathrm{N}}\left[u_{n}\right]_{\Sigma^{\varepsilon}} n+K_{\mathrm{T}} \sum_{i=1}^{2}\left[u_{\tau^{i}}\right]_{\Sigma^{\varepsilon}} \tau^{i}\right) \cdot\left(v_{1}-v_{0}\right) \mathrm{d} S(x)
\end{aligned}
$$

and the right-hand side of $(3)$ as a mapping $F: \mathcal{W}_{\mathrm{d}}\left(\Omega^{\varepsilon}\right) \rightarrow \mathbb{R}$,

$$
F(v)=\int_{\Omega^{\varepsilon}} f^{\varepsilon} \cdot v \mathrm{~d} x+\int_{\Gamma_{2}} g \cdot v_{1} \mathrm{~d} S(x) .
$$

First of all, we prove that $a$ is a continuous coercive bilinear form. The linearity in both components is clear. It is easy to compute that $a$ is coercive using the properties of $A^{\varepsilon}$ and the splitting of $u_{0}$ and $u_{1}$ in normal and tangential part. The continuity can be shown with the help of the boundedness of $A^{\varepsilon}$ and Hölder's inequality. It remains to prove that $F$ is linear and continuous. The linearity is clear. Let $v \in \mathcal{W}_{\mathrm{d}}\left(\Omega^{\varepsilon}\right)$. Using the same estimate as in (6) for $v_{0}$ and (5) for $v_{1}$, we obtain

$$
\begin{aligned}
& \left\|v_{0}\right\|_{\left[L^{2}\left(\Omega_{0}^{\varepsilon}\right)\right]^{3}}^{2} \leq C\|v\|_{\mathcal{W}_{\mathrm{d}}\left(\Omega^{\varepsilon}\right)}^{2}, \\
& \left\|v_{1}\right\|_{\left[L^{2}\left(\Omega_{1}^{\varepsilon}\right)\right]^{3}}^{2} \leq C\left\|e\left(v_{1}\right)\right\|_{\left[L^{2}\left(\Omega_{1}^{\varepsilon}\right)\right]^{3 \times 3}}^{2} \leq C\|v\|_{\mathcal{W}_{\mathrm{d}}\left(\Omega^{\varepsilon}\right)} .
\end{aligned}
$$

Thus, Hölder's inequality leads to

$$
|F(v)| \leq C\left\|f^{\varepsilon}\right\|_{\left[L^{2}\left(\Omega^{\varepsilon}\right)\right]^{3}}\|v\|_{\mathcal{W}_{\mathrm{d}}\left(\Omega^{\varepsilon}\right)}+\|g\|_{\left[L^{2}\left(\Gamma_{2}\right)\right]^{3}}\left\|v_{1}\right\|_{\left[L^{2}\left(\Gamma_{2}\right)\right]^{3}} .
$$


It remains to estimate $\left\|v_{1}\right\|_{\left[L^{2}\left(\Gamma_{2}\right)\right]^{3}}^{2}$ in $\left(90\right.$ by the norm $\|v\|_{\mathcal{W}_{d}\left(\Omega^{\varepsilon}\right)}$. We use the extension operator $P_{\varepsilon}$ from (4) together with the trace operator and (5) to receive

$$
\left\|v_{1}\right\|_{\left[L^{2}\left(\Gamma_{2}\right)\right]^{3}}^{2} \leq C\left\|P_{\varepsilon} v_{1}\right\|_{\left[H^{1}(\Omega)\right]^{3}}^{2} \leq C\left\|v_{1}\right\|_{\left[H^{1}\left(\Omega_{1}^{\varepsilon}\right)\right]^{3}}^{2} \leq C\left\|e\left(v_{1}\right)\right\|_{\left[L^{2}\left(\Omega_{1}^{\varepsilon}\right)\right]^{3 \times 3}} \leq C\|v\|_{\mathcal{W}_{\mathrm{d}}\left(\Omega^{\varepsilon}\right)}^{2} .
$$

We receive

$$
|F(v)| \leq C\left(\left\|f^{\varepsilon}\right\|_{\left[L^{2}\left(\Omega^{\varepsilon}\right)\right]^{3}}+\|g\|_{\left[L^{2}\left(\Gamma_{2}\right)\right]^{3}}\right)\|v\|_{\mathcal{W}_{\mathrm{d}}\left(\Omega^{\varepsilon}\right)} .
$$

Therefore, all assumptions of the Lax-Milgram theorem are fulfilled and we get the existence and uniqueness of the solution.

In [4] and [22], a similar approach to the proof of existence of solutions was chosen in the scalar case, where Poincaré's inequality instead of Korn's inequality is used. The following two theorems show the uniform boundedness of the weak solutions with respect to two different norms.

Theorem 3.4. Let $u^{\varepsilon} \in \mathcal{W}_{\mathrm{d}}\left(\Omega^{\varepsilon}\right)$ be the weak solution of $(3)$ and $\left\|f^{\varepsilon}\right\|_{\left[L^{2}\left(\Omega^{\varepsilon}\right)\right]^{3}} \leq \tilde{C}$ for an $\varepsilon$ independent constant $\tilde{C}$. Then, there exists an $\varepsilon$-independent constant $C$ with

$$
\left\|u^{\varepsilon}\right\|_{\mathcal{W}_{\mathrm{d}}\left(\Omega^{\varepsilon}\right)} \leq C
$$

for all admissible $0<\varepsilon \leq 1$.

Proof. We use the estimates in the proof of Theorem 3.3 with $v=u^{\varepsilon}$ to get

$$
\min \left\{\alpha, K_{\mathrm{N}}, K_{\mathrm{T}}\right\}\left\|u^{\varepsilon}\right\|_{\mathcal{W}_{\mathrm{d}}\left(\Omega^{\varepsilon}\right)}^{2} \leq a\left(u^{\varepsilon}, u^{\varepsilon}\right)=F\left(u^{\varepsilon}\right) \leq C\left(\left\|f^{\varepsilon}\right\|_{\left[L^{2}\left(\Omega^{\varepsilon}\right)\right]^{3}}+\|g\|_{\left[L^{2}\left(\Gamma_{2}\right)\right]^{3}}\right)\left\|u^{\varepsilon}\right\|_{\mathcal{W}_{\mathrm{d}}\left(\Omega^{\varepsilon}\right)},
$$

which proves that $\left\|u^{\varepsilon}\right\|_{\mathcal{W}_{\mathrm{d}}\left(\Omega^{\varepsilon}\right)} \leq \tilde{C}$ for a constant $\tilde{C}>0$ independent of $\varepsilon$.

Theorem 3.5. With the same assumption as in Theorem 3.4, there holds

$$
\left\|u^{\varepsilon}\right\|_{\left[L^{2}\left(\Omega^{\varepsilon}\right)\right]^{3}}^{2}+\left\|e\left(u_{0}^{\varepsilon}\right)\right\|_{\left[L^{2}\left(\Omega_{0}^{\varepsilon}\right)\right]^{3 \times 3}}^{2}+\left\|e\left(u_{1}^{\varepsilon}\right)\right\|_{\left[L^{2}\left(\Omega_{1}^{\varepsilon}\right)\right]^{3 \times 3}}^{2} \leq \tilde{C}
$$

for a constant $\tilde{C}>0$ independent of $\varepsilon$.

Proof. If we choose $v=u^{\varepsilon}$ in the estimates (7) and (8) and note that the constants there are independent of $\varepsilon$ we get the desired result.

\subsection{Existence result for connected case}

In the connected case, we do not have to restrict the solution space as much as in the disconnected case. Therefore, we define the solution space for the connected case as

$$
\mathcal{W}_{\mathrm{c}}\left(\Omega^{\varepsilon}\right)=\left\{u \in\left[L^{2}\left(\Omega^{\varepsilon}\right)\right]^{3}: u_{0} \in\left[H^{1}\left(\Omega_{0}^{\varepsilon}, \Gamma_{1} \cap \partial \Omega_{0}^{\varepsilon}\right)\right]^{3}, u_{1} \in\left[H^{1}\left(\Omega_{1}^{\varepsilon}, \Gamma_{1} \cap \partial \Omega_{1}^{\varepsilon}\right)\right]^{3}\right\} .
$$

Using [19, Theorem 4.4], which states Korn's inequality for a constant independent of $\varepsilon$, the space $\mathcal{W}_{\mathrm{c}}\left(\Omega^{\varepsilon}\right)$ endowed with the norm

$$
\|u\|_{\mathcal{W}_{\mathrm{c}}\left(\Omega^{\varepsilon}\right)}^{2}=\left\|e\left(u_{0}\right)\right\|_{\left[L^{2}\left(\Omega_{0}^{\varepsilon}\right)\right]^{3 \times 3}}^{2}+\left\|e\left(u_{1}\right)\right\|_{\left[L^{2}\left(\Omega_{1}^{\varepsilon}\right)\right]^{3 \times 3}}^{2}+\varepsilon\left\|[u]_{\Sigma^{\varepsilon}}\right\|_{\left[L^{2}\left(\Sigma^{\varepsilon}\right)\right]^{3}}^{2}
$$

becomes a Banach space. Note that the norms on $\mathcal{W}_{\mathrm{d}}\left(\Omega_{\varepsilon}\right)$ and $\mathcal{W}_{\mathrm{c}}\left(\Omega_{\varepsilon}\right)$ are identical. 
Theorem 3.6. Let $f^{\varepsilon} \in\left[L^{2}\left(\Omega^{\varepsilon}\right)\right]^{3}, g \in\left[L^{2}\left(\Gamma_{2}\right)\right]^{3}$. Then there exists a unique solution $u^{\varepsilon} \in$ $\mathcal{W}_{\mathrm{c}}\left(\Omega^{\varepsilon}\right)$ of (3) for all admissible $0<\varepsilon \leq 1$.

Proof. We prove this statement via Lax-Milgram theorem like in Theorem 3.3. Let $\varepsilon>0$ and the mapping $a$ be defined as in Theorem 3.3. The same proof shows that $a$ is a coercive continuous bilinear form. The right-hand side of (3) is slightly different from before, so we define the mapping $F: \mathcal{W}_{\mathrm{c}}\left(\Omega^{\varepsilon}\right) \rightarrow \mathbb{R}$

$$
F(v)=\int_{\Omega^{\varepsilon}} f^{\varepsilon} \cdot v \mathrm{~d} x+\int_{\Gamma_{2} \cap \partial \Omega_{0}^{\varepsilon}} g \cdot v_{0} \mathrm{~d} S(x)+\int_{\Gamma_{2} \cap \partial \Omega_{1}^{\varepsilon}} g \cdot v_{1} \mathrm{~d} S(x) .
$$

We have to prove that $F \in \mathcal{W}_{\mathrm{c}}\left(\Omega^{\varepsilon}\right)^{\prime}$, i.e. $F$ is linear and continuous. The linearity is clear. Let $v \in \mathcal{W}_{\mathrm{c}}\left(\Omega^{\varepsilon}\right)$. Using Hölder's inequality and Korn's inequality ([19, Theorem 4.4] applied on $\Omega_{0}^{\varepsilon}$ and $\left.\Omega_{1}^{\varepsilon}\right)$, we obtain

$$
\left|\int_{\Omega^{\varepsilon}} f^{\varepsilon} \cdot v \mathrm{~d} x\right| \leq C\left\|f^{\varepsilon}\right\|_{\left[L^{2}\left(\Omega^{\varepsilon}\right)\right]^{3}}\left(\left\|e\left(v_{0}\right)\right\|_{\left[L^{2}\left(\Omega_{0}^{\varepsilon}\right)\right]^{3 \times 3}}^{2}+\left\|e\left(v_{1}\right)\right\|_{\left[L^{2}\left(\Omega_{1}^{\varepsilon}\right)\right]^{3 \times 3}}^{2}\right)^{\frac{1}{2}} .
$$

Let $\alpha=0$ or 1 . We apply Hölder's inequality to get

$$
\left|\int_{\Gamma_{2} \cap \partial \Omega_{\alpha}^{\varepsilon}} g \cdot v_{\alpha} \mathrm{d} S(x)\right| \leq\|g\|_{\left[L^{2}\left(\Gamma_{2}\right)\right]^{3}}\left\|v_{\alpha}\right\|_{\left[L^{2}\left(\Gamma_{2} \cap \partial \Omega_{\alpha}^{\varepsilon}\right)\right]^{3}}
$$

In order to estimate the boundary terms, we use the extension operator $L_{\alpha}^{\varepsilon}$ from $\Omega_{\alpha}^{\varepsilon}$ to $\Omega$ defined in [19, Theorem 3.4]. Therefore, we can apply the trace operator on $L_{\alpha}^{\varepsilon} v_{\alpha}$, use the estimates of the trace operator and the Korn's inequality ([19, Theorem 4.4]) to get the desired estimate

$$
\left\|v_{\alpha}\right\|_{\left[L^{2}\left(\Gamma_{2} \cap \partial \Omega_{\alpha}^{\varepsilon}\right)\right]^{3}} \leq C\left\|L_{\alpha}^{\varepsilon}\left(v_{\alpha}\right)\right\|_{\left[H^{1}(\Omega)\right]^{3}} \leq C\left\|v_{\alpha}\right\|_{\left[H^{1}\left(\Omega_{\alpha}^{\varepsilon}\right)\right]^{3}} \leq C\left\|e\left(v_{\alpha}\right)\right\|_{\left[L^{2}\left(\Omega_{\alpha}^{\varepsilon}\right]^{3 \times 3}\right.} .
$$

Summarizing the previous estimates, we get the continuity of the mapping $F$

$$
|F(v)| \leq C\left(\left\|f^{\varepsilon}\right\|_{\left[L^{2}\left(\Omega^{\varepsilon}\right)\right]^{3 \times 3}}+\|g\|_{\left[L^{2}\left(\Gamma_{2}\right)\right]^{3}}\right)\|v\|_{\mathcal{W}_{\mathrm{c}}\left(\Omega^{\varepsilon}\right)} .
$$

Lax-Milgram Theorem guarantees the existence and uniqueness of the solution.

Theorem 3.7. Let $u^{\varepsilon} \in \mathcal{W}_{\mathrm{c}}\left(\Omega^{\varepsilon}\right)$ be the weak solutions of (3) and $\left\|f^{\varepsilon}\right\|_{\left[L^{2}\left(\Omega^{\varepsilon}\right)\right]^{3}} \leq \tilde{C}$ for an $\varepsilon$ independent constant $\tilde{C}$. Then, exists a constant $C$ independent of $\varepsilon$ such that

$$
\left\|u^{\varepsilon}\right\|_{\mathcal{W}_{\mathrm{c}}\left(\Omega^{\varepsilon}\right)} \leq C
$$

for all admissible $0<\varepsilon \leq 1$.

Proof. The proof is analogous to the proof of Theorem 3.4.

\section{Periodic homogenization}

This section is devoted to the rigorous upscaling of the problem in the disconnected subsection 4.2) and connected case (subsection 4.3). We mainly use the periodic unfolding method, which is briefly introduced together with some standard results for convenience first subsection 4.1). 


\subsection{Introduction to periodic unfolding method}

The periodic unfolding method was formally introduced by Doina Cioranescu, Alain Damlamian und George Griso in 2002. The main idea is to write the function, which is defined on $\Omega^{\varepsilon}$, as a function of micro- and macro-scale, which makes it easier to work with perforated domains which change with $\varepsilon$. We refer to [8], 23] and [5] for the definitions and theorems in this section and further results. The first paper considers a fixed domain, the second a perforated domain with connected or disconnected holes and the third a domain consisting of two components.

To define the periodic unfolding operator $\mathcal{T}_{i}^{\varepsilon}, i=0$ or 1 , we need another representation of $x \in \Omega$. Let $x \in \mathbb{R}^{3}$. We denote by $[x]$ the unique linear combination of the integers $\xi_{j} \in \mathbb{Z}$ and the periodicity vectors $b_{j} \in \mathbb{R}^{3}$, which are in our case the unit vectors $e_{j}$ for $j=1,2,3$, i.e. $[x]=\sum_{j=1}^{3} \xi_{j} b_{j}$, such that $\{x\}:=x-[x] \in Y$. Therefore we can rewrite $x \in \mathbb{R}^{3}$ via

$$
x=\varepsilon\left(\left[\frac{x}{\varepsilon}\right]+\left\{\frac{x}{\varepsilon}\right\}\right) .
$$

Definition 4.1. Let $i=0$ or 1 . For a Lebesgue-measurable function $\phi$ on $\Omega_{i}^{\varepsilon}$, the periodic unfolding operator $\mathcal{T}_{i}^{\varepsilon}$ is defined as

$$
\mathcal{T}_{i}^{\varepsilon}(\phi)(x, y):= \begin{cases}\phi\left(\varepsilon\left[\frac{x}{\varepsilon}\right]+\varepsilon y\right) & \text { for a.e. }(x, y) \in \hat{\Omega}^{\varepsilon} \times Y_{i}, \\ 0 & \text { for a.e. }(x, y) \in \Pi^{\varepsilon} \times Y_{i},\end{cases}
$$

where $\hat{\Omega}^{\varepsilon}=\bigcup_{\xi \in \Lambda^{\varepsilon}} \varepsilon(\xi+Y)$ and $\Pi^{\varepsilon}=\Omega \backslash \hat{\Omega}^{\varepsilon}$.

Due to our assumption, there holds $\hat{\Omega}^{\varepsilon}=\Omega^{\varepsilon}$ and $\Pi^{\varepsilon}=\emptyset$ for admissible $\varepsilon$. Note that the definition of the periodic unfolding operator does not require $\Omega$ to be representable as a union of axis-parallel cuboids. We denote by $\tilde{\tilde{f}}$ the extension of the function $f$ to $\Omega$ by zero and by $\mathcal{M}_{\mathcal{O}}(\phi)=\frac{1}{|\mathcal{O}|} \int_{\mathcal{O}} \phi \mathrm{d} y$ the mean value of the function $\phi$ over the domain $\mathcal{O}$, where $\mathcal{O}$ is a set with finite measure. We summarize a number of well-known results on periodic unfolding in our notation for future reference.

Proposition 4.2. The operator $\mathcal{T}_{i}^{\varepsilon}: L^{2}\left(\Omega_{i}^{\varepsilon}\right) \rightarrow L^{2}\left(\Omega \times Y_{i}\right), i=0,1$, is linear and continuous and

(i) $\mathcal{T}_{i}^{\varepsilon}(v w)=\mathcal{T}_{i}^{\varepsilon}(v) \mathcal{T}_{i}^{\varepsilon}(w)$ for all Lebesgue-measurable functions $v, w$ in $\Omega_{i}^{\varepsilon}$,

(ii) $\frac{1}{|Y|} \int_{\Omega \times Y_{i}} \mathcal{T}_{i}^{\varepsilon}(\phi)(x, y) \mathrm{d} x \mathrm{~d} y=\int_{\hat{\Omega}_{i}^{\varepsilon}} \phi(x) \mathrm{d} x=\int_{\Omega_{i}^{\varepsilon}} \phi(x) \mathrm{d} x-\int_{\Pi_{i}^{\varepsilon}} \phi(x) \mathrm{d} x$ for all $\phi \in L^{2}\left(\Omega_{i}^{\varepsilon}\right)$,

(iii) $\left\|\mathcal{T}_{i}^{\varepsilon}(\phi)\right\|_{L^{2}\left(\Omega \times Y_{i}\right)} \leq|Y|^{\frac{1}{2}}\|\phi\|_{L^{2}\left(\Omega_{i}^{\varepsilon}\right)}$ for all $\phi \in L^{2}\left(\Omega_{i}^{\varepsilon}\right)$,

(iv) $\mathcal{T}_{i}^{\varepsilon}(\phi) \rightarrow \phi$ strongly in $L^{2}\left(\Omega \times Y_{i}\right)$ for all $\phi \in L^{2}(\Omega)$,

(v) if $\left\{\phi^{\varepsilon}\right\}$ is a sequence in $L^{2}(\Omega)$ with $\phi^{\varepsilon} \rightarrow \phi$ strongly in $L^{2}(\Omega)$, then $\mathcal{T}_{i}^{\varepsilon}\left(\phi^{\varepsilon}\right) \rightarrow \phi$ strongly in $L^{2}\left(\Omega \times Y_{i}\right)$,

(vi) if $\phi \in L^{2}\left(Y_{i}\right) Y$-periodic and $\phi^{\varepsilon}(x)=\phi\left(\frac{x}{\varepsilon}\right)$, then $\mathcal{T}_{i}^{\varepsilon}\left(\phi^{\varepsilon}\right) \rightarrow \phi$ strongly in $L^{2}\left(\Omega \times Y_{i}\right)$,

(vii) if $\phi^{\varepsilon} \in L^{2}\left(\Omega_{i}^{\varepsilon}\right)$ with $\left\|\phi^{\varepsilon}\right\|_{L^{2}\left(\Omega_{i}^{\varepsilon}\right)} \leq C$ and $\mathcal{T}_{i}^{\varepsilon}\left(\phi^{\varepsilon}\right) \rightarrow \phi$ weakly in $L^{2}\left(\Omega \times Y_{i}\right)$, then $\widetilde{\widetilde{\phi}}^{\varepsilon} \rightarrow$ $\frac{\left|Y_{i}\right|}{|Y|} \mathcal{M}_{Y_{i}}(\phi)$ weakly in $L^{2}(\Omega)$,

(viii) if $\phi \in H^{1}\left(\Omega_{i}^{\varepsilon}\right)$, then $\nabla_{y}\left[\mathcal{T}_{i}^{\varepsilon}(\phi)\right]=\varepsilon \mathcal{T}_{i}^{\varepsilon}(\nabla \phi)$ and $\mathcal{T}_{i}^{\varepsilon}(\phi) \in L^{2}\left(\Omega, H^{1}\left(Y_{i}\right)\right)$.

The next result shows the equivalence between classic two-scale convergence of a sequence [10, 11] and the weak convergence of the unfolded sequence. 
Theorem 4.3. Let $\left\{\phi^{\varepsilon}\right\}$ be a bounded sequence in $L^{2}(\Omega)$. The following assertions are equivalent:

(i) $\left\{\mathcal{T}^{\varepsilon}\left(\phi^{\varepsilon}\right)\right\}$ converges weakly to $\phi$ in $L^{2}(\Omega \times Y)$,

(ii) $\left\{\phi^{\varepsilon}\right\}$ two-scale converges to $\phi$, which we denote by $\phi^{\varepsilon} \stackrel{2}{\longrightarrow} \phi$ for convenience.

Due to the equivalence, we often use assertions (i) and (ii) of Theorem 4.3 synonymously.

Remark 4.4. The results are still true for vector- and matrix-valued functions, if we use the period unfolding operator for every component and the standard inner product for vectors and the Frobenius inner product for matrices.

\subsection{Homogenization in the disconnected case}

In section 4.2.1, we prove some general compactness results via the periodic unfolding method, which we apply in section 4.2 .2 to derive the homogenized problem for the disconnected case.

\subsubsection{Compactness results for the disconnected case}

In the first theorem, we compute the limit of a sequence $\left\{u_{1}^{\varepsilon}\right\}$ in $\left[H^{1}\left(\Omega_{1}^{\varepsilon}, \Gamma_{1}\right)\right]^{3}$, for which we have to define the Hilbert space

$$
\left[L^{2}\left(\Omega, H_{\mathrm{per}, 0}^{1}\left(Y_{1}\right)\right)\right]^{3}:=\left\{u \in\left[L^{2}\left(\Omega, H_{\mathrm{per}}^{1}\left(Y_{1}\right)\right)\right]^{3}: \mathcal{M}_{Y_{1}}(u)=0\right\} .
$$

Theorem 4.5. Let $\left\{u_{1}^{\varepsilon}\right\}$ be a sequence in $\left[H^{1}\left(\Omega_{1}^{\varepsilon}, \Gamma_{1}\right)\right]^{3}$ with

$$
\left\|u_{1}^{\varepsilon}\right\|_{\left[L^{2}\left(\Omega_{1}^{\varepsilon}\right)\right]^{3}}+\left\|e\left(u_{1}^{\varepsilon}\right)\right\|_{\left[L^{2}\left(\Omega_{1}^{\varepsilon}\right)\right]^{3 \times 3}} \leq C
$$

for a constant $C$ independent of $\varepsilon$. Then, there exist a subsequence (again denoted by $\varepsilon$ ), $u_{1} \in$ $\left[H^{1}\left(\Omega, \Gamma_{1}\right)\right]^{3}$ and $\hat{u}_{1} \in\left[L^{2}\left(\Omega, H_{\mathrm{per}, 0}^{1}\left(Y_{1}\right)\right)\right]^{3}$ such that

$$
\begin{gathered}
\mathcal{T}_{1}^{\varepsilon}\left(u_{1}^{\varepsilon}\right) \rightarrow u_{1} \text { weakly in }\left[L^{2}\left(\Omega, H^{1}\left(Y_{1}\right)\right)\right]^{3} \\
\mathcal{T}_{1}^{\varepsilon}\left(e\left(u_{1}^{\varepsilon}\right)\right) \rightarrow e\left(u_{1}\right)+e_{y}\left(\hat{u}_{1}\right) \text { weakly in }\left[L^{2}\left(\Omega \times Y_{1}\right)\right]^{3 \times 3}
\end{gathered}
$$

Proof. The sequence $\left\{u_{1}^{\varepsilon}\right\}$ is bounded in $\left[H^{1}\left(\Omega_{1}^{\varepsilon}\right)\right]^{3}$ because, using (5), we can estimate

$$
\left\|u_{1}^{\varepsilon}\right\|_{\left[L^{2}\left(\Omega_{1}^{\varepsilon}\right)\right]^{3}}^{2}+\left\|\nabla u_{1}^{\varepsilon}\right\|_{\left[L^{2}\left(\Omega_{1}^{\varepsilon}\right)\right]^{3 \times 3}}^{2} \leq C\left\|e\left(u_{1}^{\varepsilon}\right)\right\|_{\left[L^{2}\left(\Omega_{1}^{\varepsilon}\right)\right]^{3 \times 3}}^{2} \leq C .
$$

Since the domain $\Omega_{1}^{\varepsilon}$ is connected, we can apply [24, Theorem 4.43] to get, up to a subsequence,

$$
\begin{aligned}
\mathcal{T}_{1}^{\varepsilon}\left(u_{1}^{\varepsilon}\right) & \rightarrow u_{1} \text { weakly in }\left[L^{2}\left(\Omega, H^{1}\left(Y_{1}\right)\right)\right]^{3}, \\
\mathcal{T}_{1}^{\varepsilon}\left(\nabla u_{1}^{\varepsilon}\right) & \rightarrow \nabla u_{1}+\nabla_{y} \hat{u}_{1} \text { weakly in }\left[L^{2}\left(\Omega \times Y_{1}\right)\right]^{3 \times 3}
\end{aligned}
$$

for some $u_{1} \in\left[H^{1}\left(\Omega, \Gamma_{1}\right)\right]^{3}$ and $\hat{u}_{1} \in\left[L^{2}\left(\Omega, H_{\mathrm{per}, 0}^{1}\left(Y_{1}\right)\right)\right]^{3}$. The definition of the linear strain tensor $e\left(u_{1}^{\varepsilon}\right)=\frac{1}{2}\left(\nabla u_{1}^{\varepsilon}+\left(\nabla u_{1}^{\varepsilon}\right)^{T}\right)$ directly yields

$$
\mathcal{T}_{1}^{\varepsilon}\left(e\left(u_{1}^{\varepsilon}\right)\right) \rightarrow e\left(u_{1}\right)+e_{y}\left(\hat{u}_{1}\right) \text { weakly in }\left[L^{2}\left(\Omega \times Y_{1}\right)\right]^{3 \times 3} .
$$


In the rest of the section, we want to derive the limit of a sequence $\left\{u_{0}^{\varepsilon}\right\}$ in the Hilbert space

$$
\left[\tilde{H}^{1}\left(\Omega_{0}^{\varepsilon}\right)\right]^{3}:=\left\{u \in\left[H^{1}\left(\Omega_{0}^{\varepsilon}\right)\right]^{3}: \nabla \times u=0 \text { a.e. in } \Omega_{0}^{\varepsilon}\right\} .
$$

The next lemma states the connection between the curl and symmetric gradient of some function $\phi$ and of the unfolded function $\mathcal{T}^{\varepsilon}(\phi)$.

Lemma 4.6. Let $i=0$ or 1 and $\phi \in\left[H^{1}\left(\Omega_{i}^{\varepsilon}\right)\right]^{3}$. Then, there holds

(i) $\nabla_{y} \times \mathcal{T}_{i}^{\varepsilon}(\phi)=\varepsilon \mathcal{T}_{i}^{\varepsilon}(\nabla \times \phi)$,

(ii) $e_{y}\left(\mathcal{T}_{i}^{\varepsilon}(\phi)\right)=\varepsilon \mathcal{T}_{i}^{\varepsilon}(e(\phi))$.

Proof. These are simple computations using the linearity of $\mathcal{T}_{i}^{\varepsilon}$.

The strategy of the proof of Theorem 4.7 follows [5, Theorem 2.18].

Theorem 4.7. Let $\left\{u_{0}^{\varepsilon}\right\}$ be a sequence in $\left[\tilde{H}^{1}\left(\Omega_{0}^{\varepsilon}\right)\right]^{3}$ with

$$
\left\|u_{0}^{\varepsilon}\right\|_{\left[L^{2}\left(\Omega_{0}^{\varepsilon}\right)\right]^{3}}+\left\|e\left(u_{0}^{\varepsilon}\right)\right\|_{\left[L^{2}\left(\Omega_{0}^{\varepsilon}\right)\right]^{3 \times 3}} \leq C
$$

for a constant $C$ independent of $\varepsilon$. Then, there exists a subsequence (again denoted by $\varepsilon$ ) and $u_{0} \in\left[L^{2}(\Omega)\right]^{3}$ such that

$$
\begin{gathered}
\mathcal{T}_{0}^{\varepsilon}\left(u_{0}^{\varepsilon}\right) \rightarrow u_{0} \text { weakly in }\left[L^{2}\left(\Omega, H^{1}\left(Y_{0}\right)\right)\right]^{3}, \\
\varepsilon \mathcal{T}_{0}^{\varepsilon}\left(\nabla u_{0}^{\varepsilon}\right) \rightarrow 0 \text { strongly in }\left[L^{2}\left(\Omega \times Y_{0}\right)\right]^{3 \times 3}
\end{gathered}
$$

Proof. Let $\left\{u_{0}^{\varepsilon}\right\}$ be a sequence in $\left[\tilde{H}^{1}\left(\Omega_{0}^{\varepsilon}\right)\right]^{3}$, for which (11) holds. Then, there holds due to Proposition 4.2 (iii)

$$
\begin{aligned}
& \left\|\mathcal{T}_{0}^{\varepsilon}\left(u_{0}^{\varepsilon}\right)\right\|_{\left[L^{2}\left(\Omega \times Y_{0}\right)\right]^{3}} \leq|Y|^{\frac{1}{2}}\left\|u_{0}^{\varepsilon}\right\|_{\left[L^{2}\left(\Omega_{0}^{\varepsilon}\right)\right]^{3}} \leq C, \\
& \left\|\nabla_{y} \mathcal{T}_{0}^{\varepsilon}\left(u_{0}^{\varepsilon}\right)\right\|_{\left[L^{2}\left(\Omega \times Y_{0}\right)\right]^{3 \times 3}}=\varepsilon\left\|\mathcal{T}_{0}^{\varepsilon}\left(e\left(u_{0}^{\varepsilon}\right)\right)\right\|_{\left[L^{2}\left(\Omega \times Y_{0}\right)\right]^{3 \times 3}} \leq \varepsilon|Y|^{\frac{1}{2}}\left\|e\left(u_{0}^{\varepsilon}\right)\right\|_{\left[L^{2}\left(\Omega_{0}^{\varepsilon}\right)\right]^{3 \times 3}} \leq \varepsilon C .
\end{aligned}
$$

Thus, $\mathcal{T}_{0}^{\varepsilon}\left(u_{0}^{\varepsilon}\right)$ is bounded in $\left[L^{2}\left(\Omega, H^{1}\left(Y_{0}\right)\right)\right]^{3}$. Since $\left[L^{2}\left(\Omega, H^{1}\left(Y_{0}\right)\right)\right]^{3}$ is a Hilbert space, there exists a subsequence (again denoted by $\varepsilon$ ) and $u_{0} \in\left[L^{2}\left(\Omega, H^{1}\left(Y_{0}\right)\right]^{3}\right.$ such that

$$
\begin{aligned}
\mathcal{T}_{0}^{\varepsilon}\left(u_{0}^{\varepsilon}\right) & \rightarrow u_{0} \text { weakly in }\left[L^{2}\left(\Omega, H^{1}\left(Y_{0}\right)\right)\right]^{3}, \\
\nabla_{y}\left(\mathcal{T}_{0}^{\varepsilon}\left(u_{0}^{\varepsilon}\right)\right) & \rightarrow 0 \text { strongly in }\left[L^{2}\left(\Omega \times Y_{0}\right)\right]^{3 \times 3},
\end{aligned}
$$

which yield

$$
\nabla_{y} u_{0}=0 \text { a.e. in } \Omega \times Y_{0} .
$$

This proves that $u_{0}$ is independent of $y$.

We can even derive from $\nabla \times u_{0}^{\varepsilon}=0$ that the limit satisfies $\nabla \times u_{0}=0$. 
Theorem 4.8. Let $\left\{u_{0}^{\varepsilon}\right\}$ be a bounded sequence in $\left[\tilde{H}^{1}\left(\Omega_{0}^{\varepsilon}\right)\right]^{3}$ with

$$
\mathcal{T}_{0}^{\varepsilon}\left(u_{0}^{\varepsilon}\right) \rightarrow u_{0} \text { weakly in }\left[L^{2}\left(\Omega, H^{1}\left(Y_{0}\right)\right)\right]^{3}
$$

for some $u_{0} \in\left[L^{2}(\Omega)\right]^{3}$. Then, the weak limit satisfies $\nabla \times u_{0}=0$.

Proof. Let $u_{0}^{\varepsilon}$ be as in the assumption and $\varphi(x, y)=\left(\psi_{i}(x) \eta_{i}(y)\right)_{1 \leq i \leq 3} \in\left[\mathcal{D}(\Omega) \otimes H^{1}\left(Y_{0}\right)\right]^{3}$. We write $\varphi^{\varepsilon}:=\left(\psi_{i} \eta_{i}^{\varepsilon}\right)_{1 \leq i \leq 3}:=\varphi\left(x, \frac{x}{\varepsilon}\right)$. Since

$$
\mathcal{T}_{0}^{\varepsilon}\left(\varphi_{i}^{\varepsilon}\right)=\mathcal{T}_{0}^{\varepsilon}\left(\psi_{i}\right) \mathcal{T}_{0}^{\varepsilon}\left(\eta_{i}^{\varepsilon}\right)=\mathcal{T}_{0}^{\varepsilon}\left(\psi_{i}\right) \eta_{i} \longrightarrow \psi_{i} \eta_{i} \quad \text { strongly in } L^{2}\left(\Omega \times Y_{0}\right)
$$

there holds

$$
\int_{\Omega_{0}^{\varepsilon}} u_{0}^{\varepsilon} \cdot \varphi^{\varepsilon} \mathrm{d} x=\int_{\Omega \times Y_{0}} \mathcal{T}_{0}^{\varepsilon}\left(u_{0}^{\varepsilon}\right) \cdot\left(\mathcal{T}_{0}^{\varepsilon}\left(\psi_{i}\right) \mathcal{T}_{0}^{\varepsilon}\left(\eta_{i}\right)\right)_{1 \leq i \leq 3} \mathrm{~d} y \mathrm{~d} x \longrightarrow \int_{\Omega \times Y_{0}} u_{0} \cdot \varphi \mathrm{d} y \mathrm{~d} x .
$$

Clearly, $\varphi^{\varepsilon} \in\left[H^{1}\left(\Omega_{0}^{\varepsilon}\right)\right]^{3}$. Thus, the trace of $\mathcal{T}_{0}^{\varepsilon}\left(\varphi^{\varepsilon}\right)$ with respect to $\partial Y_{0}$ exists for all $\varepsilon>0$ and we can apply the trace theorem to calculate

$$
\left\|\mathcal{T}_{0}^{\varepsilon}\left(\varphi^{\varepsilon}\right)-\varphi\right\|_{\left[L^{2}\left(\Omega \times \partial Y_{0}\right)\right]^{3}} \leq C\left(\left\|\mathcal{T}_{0}^{\varepsilon}\left(\varphi^{\varepsilon}\right)-\varphi\right\|_{\left[L^{2}\left(\Omega \times Y_{0}\right)\right]^{3}}+\left\|\nabla_{y}\left(\mathcal{T}_{0}^{\varepsilon}\left(\varphi^{\varepsilon}\right)-\varphi\right)\right\|_{\left[L^{2}\left(\Omega \times Y_{0}\right)\right]^{3 \times 3}}\right) .
$$

The convergences 12 and

$$
\nabla_{y} \mathcal{T}_{0}^{\varepsilon}\left(\varphi_{i}^{\varepsilon}\right)=\varepsilon \mathcal{T}_{0}^{\varepsilon}\left(\nabla \psi_{i}\right) \eta_{i}+\mathcal{T}_{0}^{\varepsilon}\left(\psi_{i}\right) \nabla_{y} \eta_{i} \longrightarrow \psi_{i} \nabla_{y} \eta_{i} \quad \text { strongly in }\left[L^{2}\left(\Omega \times Y_{0}\right)\right]^{3}
$$

yield

$$
\left\|\mathcal{T}_{0}^{\varepsilon}\left(\varphi^{\varepsilon}\right)-\varphi\right\|_{\left[L^{2}\left(\Omega \times \partial Y_{0}\right)\right]^{3}} \rightarrow 0 .
$$

Since $u_{0}^{\varepsilon} \in\left[H^{1}\left(\Omega_{0}^{\varepsilon}\right)\right]^{3}$, the traces exist and we can compute, using the convergences from above,

$$
\begin{aligned}
\int_{\partial \Omega_{0}^{\varepsilon}}\left(u_{0}^{\varepsilon} \times \varphi^{\varepsilon}\right) \cdot n\left(\frac{x}{\varepsilon}\right) \mathrm{d} S(x) & =\int_{\Omega \times \partial Y_{0}}\left(\mathcal{T}_{0}^{\varepsilon}\left(u_{0}^{\varepsilon}\right) \times \mathcal{T}_{0}^{\varepsilon}\left(\varphi^{\varepsilon}\right)\right) \cdot n(y) \mathrm{d} S(y) \mathrm{d} x \\
& \longrightarrow \int_{\Omega \times \partial Y_{0}}\left(u_{0} \times \varphi\right) \cdot n \mathrm{~d} S(y) \mathrm{d} x
\end{aligned}
$$

Now, for test functions with $\nabla_{y} \times \varphi=0$, there holds, using the identity for the divergence of a cross product and the weak convergence of $u_{0}^{\varepsilon}$,

$$
\begin{aligned}
0 & =\int_{\Omega_{0}^{\varepsilon}} \nabla \times u_{0}^{\varepsilon} \cdot \varphi^{\varepsilon} \mathrm{d} x \\
= & -\int_{\partial \Omega_{0}^{\varepsilon}}\left(u_{0}^{\varepsilon} \times \varphi^{\varepsilon}\right) \cdot n \mathrm{~d} S(x)+\int_{\Omega_{0}^{\varepsilon}} u_{0}^{\varepsilon} \cdot \nabla_{x} \times \varphi\left(x, \frac{x}{\varepsilon}\right) \mathrm{d} x \\
\underline{\underline{13}} & -\int_{\Omega \times \partial Y_{0}}\left(u_{0} \times \varphi\right) \cdot n \mathrm{~d} S(y) \mathrm{d} x+\int_{\Omega \times Y_{0}} u_{0} \cdot \nabla_{x} \times \varphi \mathrm{d} y \mathrm{~d} x \\
& =-\int_{\Omega \times Y_{0}} u_{0} \cdot \nabla_{y} \times \varphi \mathrm{d} y \mathrm{~d} x+\int_{\partial \Omega \times Y_{0}}\left(u_{0} \times \varphi\right) \cdot n \mathrm{~d} y \mathrm{~d} S(x)+\int_{\Omega \times Y_{0}} \nabla_{x} \times u_{0} \cdot \varphi \mathrm{d} y \mathrm{~d} x \\
& =\int_{\Omega} \nabla \times u_{0}(x) \cdot \int_{Y_{0}} \varphi(x, y) \mathrm{d} y \mathrm{~d} x .
\end{aligned}
$$


If we choose $\psi_{1}=\psi_{2}=\psi_{3}=\Psi$ for some $\Psi \in \mathcal{D}(\Omega), \eta_{2}=\eta_{3}=0$ and $\eta_{1}(y)=a y_{1}$, where $a \in \mathbb{R}^{3}$, we receive with 15 )

$$
0=\int_{\Omega} \nabla \times u_{0}(x) \int_{Y_{0}} \varphi(x, y) \mathrm{d} y \mathrm{~d} x=C \int_{\Omega}\left(\nabla \times u_{0}\right)_{1} \Psi \mathrm{d} x,
$$

which shows, due the fundamental lemma of the calculus of variations, that $\left(\nabla \times u_{0}\right)_{1}=0$. Using similar functions for the other components, we get $\nabla \times u_{0}=0$.

In the next theorem, we compute the limit of the symmetric gradient of $u_{0}^{\varepsilon}$, where the strategy of the proof follows that of [5, Theorem 2.19]. For convenience, we define the Hilbert space

$$
\left[L^{2}\left(\Omega, \tilde{H}^{1}\left(Y_{0}\right)\right)\right]^{3}:=\left\{v \in\left[L^{2}\left(\Omega, H^{1}\left(Y_{0}\right)\right)\right]^{3}: \nabla_{y} \times v=0 \text { a.e. in } \Omega \times Y_{0}\right\} .
$$

Theorem 4.9. Let $\left\{u_{0}^{\varepsilon}\right\}$ be a sequence in $\left[\tilde{H}^{1}\left(\Omega_{0}^{\varepsilon}\right)\right]^{3}$ with

$$
\left\|u_{0}^{\varepsilon}\right\|_{\left[L^{2}\left(\Omega_{0}^{\varepsilon}\right)\right]^{3}}+\left\|e\left(u_{0}^{\varepsilon}\right)\right\|_{\left[L^{2}\left(\Omega_{0}^{\varepsilon}\right)\right]^{3 \times 3}} \leq C
$$

for a constant $C$ independent of $\varepsilon$. Then, there exists a function $\hat{u}_{0} \in\left[L^{2}\left(\Omega, \tilde{H}^{1}\left(Y_{0}\right)\right)\right]^{3}$ such that up to a subsequence

$$
\begin{aligned}
Z_{0}^{\varepsilon}:=\frac{1}{\varepsilon}\left[\mathcal{T}_{0}^{\varepsilon}\left(u_{0}^{\varepsilon}\right)-\mathcal{M}_{Y_{0}}\left(\mathcal{T}_{0}^{\varepsilon}\left(u_{0}^{\varepsilon}\right)\right)\right] & \rightarrow \hat{u}_{0} \text { weakly in }\left[L^{2}\left(\Omega, \tilde{H}^{1}\left(Y_{0}\right)\right)\right]^{3}, \\
\mathcal{T}_{0}^{\varepsilon}\left(e\left(u_{0}^{\varepsilon}\right)\right) & \rightarrow e_{y}\left(\hat{u}_{0}\right) \text { weakly in }\left[L^{2}\left(\Omega \times Y_{0}\right)\right]^{3 \times 3} .
\end{aligned}
$$

Furthermore, there holds $\mathcal{M}_{Y_{0}}\left(\hat{u}_{0}\right)=0$.

Proof. Let $\left\{u_{0}^{\varepsilon}\right\}$ be a bounded sequence in $\left[\tilde{H}^{1}\left(\Omega_{0}^{\varepsilon}\right)\right]^{3}$. If we apply the Poincaré-Wirtinger inequality in $H^{1}\left(Y_{0}\right)$ and Proposition 4.2 (iii), we receive

$$
\left\|Z_{0}^{\varepsilon}\right\|_{\left[L^{2}\left(\Omega \times Y_{0}\right)\right]^{3}} \leq \frac{C}{\varepsilon}\left\|\nabla_{y} \mathcal{T}_{0}^{\varepsilon}\left(u_{0}^{\varepsilon}\right)\right\|_{\left[L^{2}\left(\Omega \times Y_{0}\right)\right]^{3 \times 3}} \leq C|Y|^{\frac{1}{2}}\left\|e\left(u_{0}^{\varepsilon}\right)\right\|_{\left[L^{2}\left(\Omega_{0}^{\varepsilon}\right)\right]^{3 \times 3}} \leq C .
$$

With Lemma 4.6 and $\nabla \times u_{0}^{\varepsilon}=0$ a.e. in $\Omega_{0}^{\varepsilon}$, we get that

$$
\nabla_{y} \times Z_{0}^{\varepsilon}=\frac{1}{\varepsilon} \nabla_{y} \times \mathcal{T}_{0}^{\varepsilon}\left(u_{0}^{\varepsilon}\right)=\mathcal{T}_{0}^{\varepsilon}\left(\nabla \times u_{0}^{\varepsilon}\right)=\mathcal{T}_{0}^{\varepsilon}(0)=0
$$

a.e. in $\Omega \times Y_{0}$. Furthermore, there holds

$$
\left\|e_{y}\left(Z_{0}^{\varepsilon}\right)\right\|_{\left[L^{2}\left(\Omega \times Y_{0}\right)\right]^{3 \times 3}}=\frac{1}{\varepsilon}\left\|e_{y}\left(\mathcal{T}_{0}^{\varepsilon}\left(u_{0}^{\varepsilon}\right)\right)\right\|_{\left[L^{2}\left(\Omega \times Y_{0}\right)\right]^{3 \times 3}}=\left\|\mathcal{T}_{0}^{\varepsilon}\left(e\left(u_{0}^{\varepsilon}\right)\right)\right\|_{\left[L^{2}\left(\Omega \times Y_{0}\right)\right]^{3 \times 3}} \leq C .
$$

Thus, the sequence $\left\{Z_{0}^{\varepsilon}\right\}$ is bounded in the Hilbert space $\left[L^{2}\left(\Omega, \tilde{H}^{1}\left(Y_{0}\right)\right)\right]^{3}$ and, therefore, there exists a function $\hat{u}_{0} \in\left[L^{2}\left(\Omega, \tilde{H}^{1}\left(Y_{0}\right)\right)\right]^{3}$ such that up to a subsequence

$$
Z_{0}^{\varepsilon} \rightarrow \hat{u}_{0} \text { weakly in }\left[L^{2}\left(\Omega, \tilde{H}^{1}\left(Y_{0}\right)\right)\right]^{3} \text {. }
$$


So

$$
\mathcal{T}_{0}^{\varepsilon}\left(e\left(u_{0}^{\varepsilon}\right)\right)=e_{y}\left(Z_{0}^{\varepsilon}\right) \rightarrow e_{y}\left(\hat{u}_{0}\right) \text { weakly in }\left[L^{2}\left(\Omega \times Y_{0}\right)\right]^{3 \times 3}
$$

and since

$$
\mathcal{M}_{Y_{0}}\left(Z_{0}^{\varepsilon}\right)=\frac{1}{\varepsilon}\left[\mathcal{M}_{Y_{0}}\left(\mathcal{T}_{0}^{\varepsilon}\left(u_{0}^{\varepsilon}\right)\right)-\mathcal{M}_{Y_{0}}\left(\mathcal{T}_{0}^{\varepsilon}\left(u_{0}^{\varepsilon}\right)\right)\right]=0
$$

for all $\varepsilon$, we receive $\mathcal{M}_{Y_{0}}\left(\hat{u}_{0}\right)=0$.

Due to Proposition 4.2 (viii), there exists the trace of the unfolding operator with respect to $y$ and we can prove the following result.

Theorem 4.10. Let $u^{\varepsilon}, \varphi \in \mathcal{W}_{\mathrm{d}}\left(\Omega^{\varepsilon}\right)$. Then, there holds

$$
\frac{1}{\varepsilon|Y|} \int_{\Omega} \int_{\Sigma_{Y}}\left(\mathcal{T}_{1}^{\varepsilon}\left(u_{1}^{\varepsilon}\right)-\mathcal{T}_{0}^{\varepsilon}\left(u_{0}^{\varepsilon}\right)\right) \cdot\left(\mathcal{T}_{1}^{\varepsilon}\left(\varphi_{1}\right)-\mathcal{T}_{0}^{\varepsilon}\left(\varphi_{0}\right)\right) \mathrm{d} S(y) \mathrm{d} x=\int_{\Sigma^{\varepsilon}}\left(u_{1}^{\varepsilon}-u_{0}^{\varepsilon}\right) \cdot\left(\varphi_{1}-\varphi_{0}\right) \mathrm{d} S(x) .
$$

Proof. The proof is similar to that of [5, Lemma 2.14].

A direct consequence is the following corollary.

Corollary 4.11. Let $u^{\varepsilon} \in \mathcal{W}_{\mathrm{d}}\left(\Omega^{\varepsilon}\right)$. Then, there holds

$$
\frac{1}{\varepsilon|Y|} \int_{\Omega} \int_{\Sigma_{Y}}\left|\mathcal{T}_{1}^{\varepsilon}\left(u_{1}^{\varepsilon}\right)-\mathcal{T}_{0}^{\varepsilon}\left(u_{0}^{\varepsilon}\right)\right|^{2} \mathrm{~d} S(y) \mathrm{d} x=\int_{\Sigma^{\varepsilon}}\left|u_{1}^{\varepsilon}-u_{0}^{\varepsilon}\right|^{2} \mathrm{~d} S(x) .
$$

Proof. The statement follows directly from Theorem 4.10, if we choose $\varphi=u^{\varepsilon}$.

\subsubsection{Passage to the limit for the disconnected case}

In this section, we apply the results proven in the section before to derive the homogenized problem. We define the Banach spaces

$$
\left[L^{2}\left(\Omega, \tilde{H}_{0}^{1}\left(Y_{0}\right)\right)\right]^{3}:=\left\{u \in\left[L^{2}\left(\Omega, H^{1}\left(Y_{0}\right)\right)\right]^{3}: \nabla_{y} \times u=0 \text { a.e. in } \Omega \times Y_{0}, \mathcal{M}_{Y_{0}}(u)=0\right\}
$$

endowed with the norm $\left(\int_{\Omega}\left\|e_{y}(u)\right\|_{\left[L^{2}\left(Y_{0}\right)\right]^{3 \times 3}}^{2} \mathrm{~d} x\right)^{\frac{1}{2}}$ and

$$
\mathcal{Z}\left(\Omega, Y_{1}, \Gamma_{1}\right):=\left[H^{1}\left(\Omega, \Gamma_{1}\right)\right]^{3} \times\left[L^{2}\left(\Omega, H_{\mathrm{per}, 0}^{1}\left(Y_{1}\right)\right)\right]^{3} \times\left[L_{\text {curl }}^{2}(\Omega)\right]^{3},
$$

whereby $\left[L_{\text {curl }}^{2}(\Omega)\right]^{3}:=\left\{u \in\left[L^{2}(\Omega)\right]^{3}: \nabla \times u=0\right.$ a.e. in $\left.\Omega\right\}$, endowed with the norm

$$
\|u\|_{\mathcal{Z}\left(\Omega, Y_{1}, \Gamma_{1}\right)}^{2}=\left\|e\left(u_{1}\right)\right\|_{\left[L^{2}(\Omega)\right]^{3 \times 3}}^{2}+\int_{\Omega}\left\|e_{y}\left(\hat{u}_{1}\right)\right\|_{\left[L^{2}\left(Y_{1}\right)\right]^{3 \times 3}}^{2} \mathrm{~d} x+\left\|u_{0}\right\|_{\left[L^{2}(\Omega)\right]^{3}}^{2}
$$

for all $u=\left(u_{1}, \hat{u}_{1}, u_{0}\right) \in \mathcal{Z}\left(\Omega, Y_{1}, \Gamma_{1}\right)$. Having introduced this notation, we can formulate the limit problem. Afterwards, we rewrite the limit problem with the help of auxiliary cell problems and show that the solution is unique. 
Theorem 4.12. Let $\left\{u^{\varepsilon}\right\}$ be a sequence of weak solutions of the problems (3) with $u^{\varepsilon} \in \mathcal{W}\left(\Omega^{\varepsilon}\right)$ and $\left\{f^{\varepsilon}\right\} \subset\left[L^{2}\left(\Omega^{\varepsilon}\right)\right]^{3}$ such that

$$
\left.\mathcal{T}_{\alpha}^{\varepsilon}\left(f^{\varepsilon}\right) \rightarrow f\right|_{\Omega \times Y_{\alpha}} \text { in }\left[L^{2}\left(\Omega \times Y_{\alpha}\right)\right]^{3}
$$

for some $f \in\left[L^{2}(\Omega \times Y)\right]^{3}$ and $\alpha=0$ or 1 . Then, there exists $u=\left(u_{1}, \hat{u}_{1}, u_{0}\right) \in \mathcal{Z}\left(\Omega, Y_{1}, \Gamma_{1}\right)$ and $\hat{u}_{0} \in\left[L^{2}\left(\Omega, \tilde{H}_{0}^{1}\left(Y_{0}\right)\right)\right]^{3}$ such that

$$
\left\{\begin{array}{l}
\mathcal{T}_{1}^{\varepsilon}\left(u_{1}^{\varepsilon}\right) \rightarrow u_{1} \text { weakly in }\left[L^{2}\left(\Omega, H^{1}\left(Y_{1}\right)\right)\right]^{3} \\
\mathcal{T}_{1}^{\varepsilon}\left(e\left(u_{1}^{\varepsilon}\right)\right) \rightarrow e\left(u_{1}\right)+e_{y}\left(\hat{u}_{1}\right) \text { weakly in }\left[L^{2}\left(\Omega \times Y_{1}\right)\right]^{3 \times 3} \\
\mathcal{T}_{0}^{\varepsilon}\left(u_{0}^{\varepsilon}\right) \rightarrow u_{0} \text { weakly in }\left[L^{2}\left(\Omega, \tilde{H}^{1}\left(Y_{0}\right)\right)\right]^{3} \\
\mathcal{T}_{0}^{\varepsilon}\left(e\left(u_{0}^{\varepsilon}\right)\right) \rightarrow e_{y}\left(\hat{u}_{0}\right) \text { weakly in }\left[L^{2}\left(\Omega \times Y_{0}\right)\right]^{3 \times 3}
\end{array}\right.
$$

where $u$ is the solution of the problem

$$
\begin{array}{rl}
\int_{\Omega} \int_{Y_{1}} & A(y)\left(e\left(u_{1}\right)+e_{y}\left(\hat{u}_{1}\right)\right)\left(e\left(v_{1}\right)+e_{y}\left(\hat{v}_{1}\right)\right) \mathrm{d} y \mathrm{~d} x \\
& +\int_{\Omega} \int_{\Sigma_{Y}}\left(K_{\mathrm{N}}\left[u_{1} \cdot n-u_{0} \cdot n\right] n+K_{\mathrm{T}} \sum_{i=1}^{2}\left[u_{1} \cdot \tau^{i}-u_{0} \cdot \tau^{i}\right] \tau^{i}\right) \cdot\left(v_{1}-v_{0}\right) \mathrm{d} S(y) \mathrm{d} x \\
& =\int_{\Omega} \int_{Y_{1}} f \mathrm{~d} y \cdot v_{1} \mathrm{~d} x+\int_{\Omega} \int_{Y_{0}} f \mathrm{~d} y \cdot v_{0} \mathrm{~d} x+\int_{\Gamma_{2}} g \cdot v_{1} \mathrm{~d} S(x)
\end{array}
$$

for all $v=\left(v_{1}, \hat{v}_{1}, v_{0}\right) \in \mathcal{Z}\left(\Omega, Y_{1}, \Gamma_{1}\right)$.

Proof. Let $\left\{u^{\varepsilon}\right\}$ be a sequence of weak solutions of problem (3) with $u^{\varepsilon} \in \mathcal{W}_{\mathrm{d}}\left(\Omega^{\varepsilon}\right)$. We apply Theorem 3.4 and Theorem 3.5 to get

$$
\left\|u^{\varepsilon}\right\|_{\left[L^{2}\left(\Omega^{\varepsilon}\right)\right]^{3}}^{2}+\left\|e\left(u_{0}^{\varepsilon}\right)\right\|_{\left[L^{2}\left(\Omega_{0}^{\varepsilon}\right)\right]^{3 \times 3}}^{2}+\left\|e\left(u_{1}^{\varepsilon}\right)\right\|_{\left[L^{2}\left(\Omega_{1}^{\varepsilon}\right]^{3 \times 3}\right.}^{2}<C .
$$

Using the theorems from section 4.2.1, the convergences (17) follow directly. We rewrite the weak formulation of problem (3) using Theorem 4.10 and Proposition 4.2 (ii) and (ii) to get the unfolded problem, whereas the integral over $\Gamma_{2} \cap \partial \Omega_{0}^{\varepsilon}$ vanishes since $\Gamma_{2} \cap \partial \Omega_{0}^{\varepsilon}=\emptyset$,

$$
\begin{aligned}
\int_{\Omega} \int_{Y_{1}} \mathcal{T}_{1}^{\varepsilon}\left(A^{\varepsilon}\right) \mathcal{T}_{1}^{\varepsilon}\left(e\left(u_{1}^{\varepsilon}\right)\right) \mathcal{T}_{1}^{\varepsilon}\left(e\left(\varphi_{1}\right)\right) \mathrm{d} y \mathrm{~d} x+\int_{\Omega} \int_{Y_{0}} \mathcal{T}_{0}^{\varepsilon}\left(A^{\varepsilon}\right) \mathcal{T}_{0}^{\varepsilon}\left(e\left(u_{0}^{\varepsilon}\right)\right) \mathcal{T}_{0}^{\varepsilon}\left(e\left(\varphi_{0}\right)\right) \mathrm{d} y \mathrm{~d} x \\
\quad+\int_{\Omega} \int_{\Sigma_{Y}}\left(K_{\mathrm{N}}\left[\mathcal{T}_{1}^{\varepsilon}\left(u_{1}^{\varepsilon} \cdot n\right)-\mathcal{T}_{0}^{\varepsilon}\left(u_{0}^{\varepsilon} \cdot n\right)\right] n\right) \cdot\left(\mathcal{T}_{1}^{\varepsilon}\left(\varphi_{1}\right)-\mathcal{T}_{0}^{\varepsilon}\left(\varphi_{0}\right)\right) \mathrm{d} S(y) \mathrm{d} x \\
\quad+\int_{\Omega} \int_{\Sigma_{Y}}\left(K_{\mathrm{T}} \sum_{i=1}^{2}\left[\mathcal{T}_{1}^{\varepsilon}\left(u_{1}^{\varepsilon} \cdot \tau^{i}\right)-\mathcal{T}_{0}^{\varepsilon}\left(u_{0}^{\varepsilon} \cdot \tau^{i}\right)\right] \tau^{i}\right) \cdot\left(\mathcal{T}_{1}^{\varepsilon}\left(\varphi_{1}\right)-\mathcal{T}_{0}^{\varepsilon}\left(\varphi_{0}\right)\right) \mathrm{d} S(y) \mathrm{d} x \\
\quad=\int_{\Omega} \int_{Y_{1}} \mathcal{T}_{1}^{\varepsilon}\left(f^{\varepsilon}\right) \cdot \mathcal{T}_{1}^{\varepsilon}\left(\varphi_{1}\right) \mathrm{d} y \mathrm{~d} x+\int_{\Omega} \int_{Y_{0}} \mathcal{T}_{0}^{\varepsilon}\left(f^{\varepsilon}\right) \cdot \mathcal{T}_{0}^{\varepsilon}\left(\varphi_{0}\right) \mathrm{d} y \mathrm{~d} x+\int_{\Gamma_{2}} g \cdot \varphi_{1} \mathrm{~d} S(x) .
\end{aligned}
$$

We choose the test functions

$$
\varphi_{0}=\varphi_{0}^{\varepsilon}:=v_{0}+\varepsilon\left(w_{i}^{0} \widehat{\psi_{i}^{0}}\right)_{1 \leq i \leq 3} \text { and } \quad \varphi_{1}=\varphi_{1}^{\varepsilon}:=v_{1}+\varepsilon\left(w_{i}^{1} \widehat{\psi_{i}^{1}}\right)_{1 \leq i \leq 3}
$$


with $v_{1} \in\left[\mathcal{D}\left(\bar{\Omega}, \Gamma_{1}\right)\right]^{3}:=\left\{\phi \in[\mathcal{D}(\bar{\Omega})]^{3}: v\right.$ is equal to 0 in a neighbourhood of $\left.\Gamma_{1}\right\}, w^{0}, w^{1}, v_{0} \in$ $[\mathcal{D}(\Omega)]^{3}, \widehat{\psi^{0}}(x)=\psi^{0}\left(\frac{x}{\varepsilon}\right)$ with $\psi^{0} \in\left[H^{1}\left(Y_{0}\right)\right]^{3}$ (Y-periodically extended to $\mathbb{R}^{3}$ ), $\widehat{\psi}^{1}(x)=\psi^{1}\left(\frac{x}{\varepsilon}\right)$ with $\psi^{1} \in\left[H_{\text {per }}^{1}\left(Y_{1}\right)\right]^{3}$ such that $\nabla \times \varphi_{0}^{\varepsilon}=0$. Then, $\varphi^{\varepsilon} \in \mathcal{W}_{\mathrm{d}}\left(\Omega^{\varepsilon}\right)$,

$$
\mathcal{T}_{0}^{\varepsilon}\left(\varphi_{0}^{\varepsilon}\right) \in\left[L^{2}\left(\Omega, \tilde{H}^{1}\left(Y_{0}\right)\right)\right]^{3} \text { and } \mathcal{T}_{1}^{\varepsilon}\left(\varphi_{1}^{\varepsilon}\right) \in\left[L^{2}\left(\Omega, H^{1}\left(Y_{1}\right)\right)\right]^{3} .
$$

Let $\alpha=0$ or 1 . Since $\mathcal{T}_{\alpha}^{\varepsilon}\left(w_{i}^{\alpha}\right) \mathcal{T}_{\alpha}^{\varepsilon}\left(\widehat{\psi_{i}^{\alpha}}\right) \rightarrow w_{i}^{\alpha} \psi_{i}^{\alpha}$ strongly in $L^{2}\left(\Omega \times Y_{\alpha}\right)$ for all $i=1,2,3$, there holds

$$
\mathcal{T}_{\alpha}^{\varepsilon}\left(\varphi_{\alpha}^{\varepsilon}\right) \rightarrow v_{\alpha} \text { strongly in }\left[L^{2}\left(\Omega \times Y_{\alpha}\right)\right]^{3} .
$$

Every component of the symmetric gradient of $\varphi_{\alpha}^{\varepsilon}$ is of the form

$$
\begin{aligned}
e_{k l}\left(\varphi_{\alpha}^{\varepsilon}\right)=e_{k l}\left(v_{\alpha}\right)+\frac{1}{2}\left[\varepsilon \partial_{x_{k}} w_{l}^{\alpha}(x) \psi_{l}^{\alpha}\left(\frac{x}{\varepsilon}\right)+w_{l}^{\alpha}(x) \partial_{y_{k}} \psi_{l}^{\alpha}\left(\frac{x}{\varepsilon}\right)\right. \\
\left.+\varepsilon \partial_{x_{l}} w_{k}^{\alpha}(x) \psi_{k}^{\alpha}\left(\frac{x}{\varepsilon}\right)+w_{k}^{\alpha}(x) \partial_{y_{l}} \psi_{k}^{\alpha}\left(\frac{x}{\varepsilon}\right)\right] .
\end{aligned}
$$

If we apply the periodic unfolding operator to $e_{k l}\left(\varphi_{\alpha}^{\varepsilon}\right)$, we get

$$
\mathcal{T}_{\alpha}^{\varepsilon}\left(e_{k l}\left(\varphi_{\alpha}^{\varepsilon}\right)\right) \rightarrow e_{k l}\left(v_{\alpha}\right)+\frac{1}{2}\left[w_{l}^{\alpha}(x) \partial_{y_{k}} \psi_{l}^{\alpha}(y)+w_{k}^{\alpha}(x) \partial_{y_{l}} \psi_{k}^{\alpha}(y)\right]=e_{k l}\left(v_{\alpha}\right)+\left(e_{y}\left(\hat{v}_{\alpha}\right)\right)_{k l}
$$

strongly in $L^{2}\left(\Omega \times Y_{\alpha}\right)$ with $\hat{v}_{\alpha}=\left(w_{i}^{\alpha}(x) \psi_{i}^{\alpha}(y)\right)_{1 \leq i \leq 3}$. So

$$
\mathcal{T}_{\alpha}^{\varepsilon}\left(e\left(\varphi_{\alpha}^{\varepsilon}\right)\right) \rightarrow e\left(v_{\alpha}\right)+e_{y}\left(\hat{v}_{\alpha}\right) \text { strongly in }\left[L^{2}\left(\Omega \times Y_{\alpha}\right)\right]^{3 \times 3} .
$$

We estimate the interface term with respect to the normal direction using Hölder's inequality, Corollary 4.11 and boundedness of the solution

$$
\begin{gathered}
\int_{\Omega} \int_{\Sigma_{Y}}\left(K_{\mathrm{N}}\left[\mathcal{T}_{1}^{\varepsilon}\left(u_{1}^{\varepsilon} \cdot n\right)-\mathcal{T}_{0}^{\varepsilon}\left(u_{0}^{\varepsilon} \cdot n\right)\right] n\right) \cdot \mathcal{T}_{0}^{\varepsilon}\left(\varepsilon\left(w_{i}^{\alpha} \widehat{\psi}_{i}^{\alpha}\right)_{1 \leq i \leq 3}\right) \mathrm{d} S(y) \mathrm{d} x \\
\leq C \varepsilon^{\frac{1}{2}}\left\|u_{1}^{\varepsilon}-u_{0}^{\varepsilon}\right\|_{L^{2}\left(\Sigma^{\varepsilon}\right)} \varepsilon\left\|\left(\mathcal{T}_{0}^{\varepsilon}\left(w_{i}^{\alpha}\right) \psi_{i}^{\alpha}\right)_{1 \leq i \leq 3}\right\|_{\left[L^{2}\left(\Omega \times \Sigma_{Y}\right)\right]^{3}} \leq C \varepsilon .
\end{gathered}
$$

Therefore, this integral converges to zero. The analogous result holds for the terms with $\tau_{i}, i=1,2$ instead of $n$. Since

$$
\mathcal{T}_{0}^{\varepsilon}\left(v_{0}\right) \rightarrow v_{0} \text { in }\left[L^{2}\left(\Omega \times Y_{0}\right)\right]^{3} \text { and } \mathcal{T}_{0}^{\varepsilon}\left(e\left(v_{0}\right)\right) \rightarrow e\left(v_{0}\right) \text { in }\left[L^{2}\left(\Omega \times Y_{0}\right)\right]^{3 \times 3}
$$

we know that $e_{y}\left(\mathcal{T}_{0}^{\varepsilon}\left(v_{0}\right)\right) \rightarrow 0$ strongly in $\left[L^{2}\left(\Omega \times Y_{0}\right)\right]^{3 \times 3}$ and thus

$$
\mathcal{T}_{0}^{\varepsilon}\left(v_{0}\right) \rightarrow v_{0} \text { strongly in }\left[L^{2}\left(\Omega, \tilde{H}^{1}\left(Y_{0}\right)\right)\right]^{3} .
$$

This yields the convergence of the traces of $\mathcal{T}_{0}^{\varepsilon}\left(v_{0}\right)$ with respect to $y$. Similarly, we receive for $v_{1}$

$$
\mathcal{T}_{1}^{\varepsilon}\left(v_{1}\right) \rightarrow v_{1} \text { strongly in }\left[L^{2}\left(\Omega, H^{1}\left(Y_{1}\right)\right)\right]^{3} .
$$


If we plug in the test function in $(19)$ and pass to the limit, we get

$$
\begin{array}{rl}
\int_{\Omega} \int_{Y_{1}} & A(y)\left(e\left(u_{1}\right)+e_{y}\left(\hat{u}_{1}\right)\right)\left(e\left(v_{1}\right)+e_{y}\left(\hat{v}_{1}\right)\right) \mathrm{d} y \mathrm{~d} x+\int_{\Omega} \int_{Y_{0}} A(y) e_{y}\left(\hat{u}_{0}\right)\left(e\left(v_{0}\right)+e_{y}\left(\hat{v}_{0}\right)\right) \mathrm{d} y \mathrm{~d} x \\
& +\int_{\Omega} \int_{\Sigma_{Y}}\left(K_{\mathrm{N}}\left[u_{1} \cdot n-u_{0} \cdot n\right] n+K_{\mathrm{T}} \sum_{i=1}^{2}\left[u_{1} \cdot \tau^{i}-u_{0} \cdot \tau^{i}\right] \tau^{i}\right) \cdot\left(v_{1}-v_{0}\right) \mathrm{d} S(y) \mathrm{d} x \\
& =\int_{\Omega} \int_{Y_{1}} f \mathrm{~d} y \cdot v_{1} \mathrm{~d} x+\int_{\Omega} \int_{Y_{0}} f \mathrm{~d} y \cdot v_{0} \mathrm{~d} x+\int_{\Gamma_{2}} g \cdot v_{1} \mathrm{~d} S(x) .
\end{array}
$$

Since $\mathcal{D}\left(\bar{\Omega}, \Gamma_{1}\right)$ is dense in $H^{1}\left(\Omega, \Gamma_{1}\right)$ and $\mathcal{D}(\Omega) \times H_{\mathrm{per}}^{1}\left(Y_{1}\right)$ is dense in $L^{2}\left(\Omega, H_{\mathrm{per}}^{1}\left(Y_{1}\right)\right)$, the homogenized problem is true for all $v_{1} \in\left[H^{1}\left(\Omega, \Gamma_{1}\right)\right]^{3}$ and $\hat{v}_{1} \in\left[L^{2}\left(\Omega, H_{\mathrm{per}, 0}^{1}\left(Y_{1}\right)\right)\right]^{3}$.

To improve this result, we choose as a test function

$$
\varphi_{1}=0 \quad \text { and } \quad \varphi_{0}=\varepsilon e\left(v_{0}(x)\right) \cdot \psi\left(\frac{x}{\varepsilon}\right)=\varepsilon e\left(v_{0}(x)\right) \cdot \frac{x}{\varepsilon}
$$

with $v_{0} \in[\mathcal{D}(\Omega)]^{3}$ with $\nabla \times v_{0}=0$ and $\psi(y)=y \in\left[H^{1}\left(Y_{0}\right)\right]^{3} Y$-periodically extended to $\mathbb{R}^{3}$. Using the fact that $\nabla \times v_{0}=0$, it is easy to compute that $\varphi^{\varepsilon} \in \mathcal{W}_{\mathrm{d}}\left(\Omega^{\varepsilon}\right)$. Therefore, we can rewrite (19)

$$
\begin{aligned}
& \int_{\Omega} \int_{Y_{0}} \mathcal{T}_{0}^{\varepsilon}\left(A^{\varepsilon}\right) \mathcal{T}_{0}^{\varepsilon}\left(e\left(u_{0}^{\varepsilon}\right)\right) \mathcal{T}_{0}^{\varepsilon}\left(e\left(\varphi_{0}\right)\right) \mathrm{d} y \mathrm{~d} x \\
& =\int_{\Omega} \int_{\Sigma_{Y}}\left(K_{\mathrm{N}}\left[\mathcal{T}_{1}^{\varepsilon}\left(u_{1}^{\varepsilon} \cdot n\right)-\mathcal{T}_{0}^{\varepsilon}\left(u_{0}^{\varepsilon} \cdot n\right)\right] n\right) \cdot \mathcal{T}_{0}^{\varepsilon}\left(\varphi_{0}\right) \mathrm{d} S(y) \mathrm{d} x \\
& \quad+\int_{\Omega} \int_{\Sigma_{Y}}\left(K_{\mathrm{T}} \sum_{i=1}^{2}\left[\mathcal{T}_{1}^{\varepsilon}\left(u_{1}^{\varepsilon} \cdot \tau^{i}\right)-\mathcal{T}_{0}^{\varepsilon}\left(u_{0}^{\varepsilon} \cdot \tau^{i}\right)\right] \tau^{i}\right) \cdot \mathcal{T}_{0}^{\varepsilon}\left(\varphi_{0}\right) \mathrm{d} S(y) \mathrm{d} x+\int_{\Omega} \int_{Y_{0}} \mathcal{T}_{0}^{\varepsilon}\left(f^{\varepsilon}\right) \cdot \mathcal{T}_{0}^{\varepsilon}\left(\varphi_{0}\right) \mathrm{d} y \mathrm{~d} x
\end{aligned}
$$

With similar argumentation as above, the right-hand side disappears for $\varepsilon \rightarrow 0$ and

$$
\mathcal{T}_{0}^{\varepsilon}\left(\varepsilon e_{k l}\left(e\left(v_{0}(x)\right) \frac{x}{\varepsilon}\right)\right) \rightarrow e_{k l}\left(v_{0}\right)(x)=\left(e_{y}\left(e\left(v_{0}(x) y\right)\right)_{k l} .\right.
$$

Thus, we obtain for all $v_{0} \in[\mathcal{D}(\Omega)]$ with $\nabla \times v_{0}=0$

$$
\int_{\Omega} \int_{Y_{0}} A(y) e_{y}\left(\hat{u}_{0}\right) e\left(v_{0}\right) \mathrm{d} y \mathrm{~d} x=0 .
$$

If we choose $v_{1}=\hat{v}_{1}=v_{0}=0$ in 20) there holds

$$
\int_{\Omega} \int_{Y_{0}} A(y) e_{y}\left(\hat{u}_{0}\right) e_{y}\left(\hat{v}_{0}\right) \mathrm{d} y \mathrm{~d} x=0 .
$$

Thus, we obtain for all $v_{1} \in\left[H^{1}\left(\Omega, \Gamma_{1}\right)\right]^{3}, \hat{v}_{1} \in\left[L^{2}\left(\Omega, H_{\mathrm{per}, 0}^{1}\left(Y_{1}\right)\right)\right]^{3}, v_{0} \in[\mathcal{D}(\Omega)]^{3}$ with $\nabla \times v_{0}=0$

$$
\begin{array}{rl}
\int_{\Omega} \int_{Y_{1}} & A(y)\left(e\left(u_{1}\right)+e_{y}\left(\hat{u}_{1}\right)\right)\left(e\left(v_{1}\right)+e_{y}\left(\hat{v}_{1}\right)\right) \mathrm{d} y \mathrm{~d} x \\
& +\int_{\Omega} \int_{\Sigma_{Y}}\left(K_{\mathrm{N}}\left[u_{1} \cdot n-u_{0} \cdot n\right] n+K_{\mathrm{T}} \sum_{i=1}^{2}\left[u_{1} \cdot \tau^{i}-u_{0} \cdot \tau^{i}\right] \tau^{i}\right) \cdot\left(v_{1}-v_{0}\right) \mathrm{d} S(y) \mathrm{d} x \\
& =\int_{\Omega} \int_{Y_{1}} f \mathrm{~d} y \cdot v_{1} \mathrm{~d} x+\int_{\Omega} \int_{Y_{0}} f \mathrm{~d} y \cdot v_{0} \mathrm{~d} x+\int_{\Gamma_{2}} g \cdot v_{1} \mathrm{~d} S(x) .
\end{array}
$$


Since $\left\{v \in[\mathcal{D}(\Omega)]^{3}: \nabla \times v=0\right.$ a.e. in $\left.\Omega\right\}$ is dense in $L_{\text {curl }}^{2}(\Omega)$, we get the desired result. Using that the solution is unique, which we prove below, all the convergences above hold true for the whole sequence.

In the next theorem, we rewrite the homogenized problem from Theorem 4.12 as a macroscopic problem with an auxiliary cell problem.

Theorem 4.13. Let $\left\{u^{\varepsilon}\right\}$ be as in Theorem 4.12. We can reformulate the homogenized problem 18) as follows: Find $u_{1} \in\left[H^{1}\left(\Omega, \Gamma_{1}\right)\right]^{3}, u_{0} \in\left[L_{\text {curl }}^{2}(\Omega)\right]^{3}$ such that

$$
\begin{aligned}
& \int_{\Omega} A_{1}^{\text {hom }} e\left(u_{1}\right) e\left(v_{1}\right) \mathrm{d} x \\
& \quad+\int_{\Omega} \int_{\Sigma_{Y}}\left(K_{\mathrm{N}}\left(u_{1} \cdot n-u_{0} \cdot n\right) n+K_{\mathrm{T}} \sum_{i=1}^{2}\left(u_{1} \cdot \tau^{i}-u_{0} \cdot \tau^{i}\right) \tau^{i}\right) \cdot\left(v_{1}-v_{0}\right) \mathrm{d} S(y) \mathrm{d} x \\
& \quad=\int_{\Omega} \int_{Y_{0}} f \mathrm{~d} y \cdot v_{0} \mathrm{~d} x+\int_{\Omega} \int_{Y_{1}} f \mathrm{~d} y \cdot v_{1} \mathrm{~d} x+\int_{\Gamma_{2}} g \cdot v_{1} \mathrm{~d} S(x)
\end{aligned}
$$

where

$$
\left(A_{1}^{\mathrm{hom}}\right)_{i j k h}=\int_{Y_{1}} a_{i j k h}(y)-\sum_{l, m=1}^{3} a_{i j l m}\left(e_{y}\left(\chi_{1}^{k h}\right)\right)_{l m} \mathrm{~d} y
$$

and $\chi_{1}^{l m} \in\left[H_{\mathrm{per}, 0}^{1}\left(Y_{1}\right)\right]^{3}, l, m \in\{1,2,3\}$, is the unique solution of

$$
\begin{cases}\left(-\sum_{j=1}^{3} \frac{\partial}{\partial y_{j}}\left[\left(A e_{y}\left(\chi_{1}^{l m}\right)\right)_{i j}-a_{i j l m}\right]\right)_{1 \leq i \leq 3}=0 & \text { in } Y_{1}, \\ \left(-\sum_{j=1}^{3}\left[\left(A e_{y}\left(\chi_{1}^{l m}\right)\right)_{i j}-a_{i j l m}\right] n_{j}\right)_{1 \leq i \leq 3}=0 & \text { on } \Sigma_{Y} .\end{cases}
$$

Proof. This result follows by standard arguments. We refer to [19] to see the steps of the proof in detail.

Similar to [17, Theorem II.1.1], it can be proven that there exist $\alpha^{\text {hom }}, \beta^{\text {hom }} \in \mathbb{R}$ with $0<$ $\alpha^{\text {hom }}<\beta^{\text {hom }}$ such that $A_{1}^{\text {hom }} \in M\left(\alpha^{\text {hom }}, \beta^{\text {hom }}, \Omega\right)$. With this fact we are able to prove the uniqueness of the solution of (21).

Theorem 4.14. The solutions $u_{1} \in\left[H^{1}\left(\Omega, \Gamma_{1}\right)\right]^{3}$ and $u_{0} \in\left[L_{\text {curl }}^{2}(\Omega)\right]^{3}$ of the macroscopic problem (21) are unique.

Proof. We assume that there exist two solutions $u=\left(u_{1}, u_{0}\right), w=\left(w_{1}, w_{0}\right) \in\left[H^{1}\left(\Omega, \Gamma_{1}\right)\right]^{3} \times$ $\left[L_{\text {curl }}^{2}(\Omega)\right]^{3}$ of the problem 21 . If we choose as test function $v_{1}=u_{1}-w_{1}$ and $v_{0}=u_{0}-w_{0}$, we can estimate, using that $A_{1}^{\text {hom }}$ is coercive and Korn's inequality for functions which are zero on part of the boundary,

$$
\begin{aligned}
0= & \int_{\Omega} A_{1}^{\text {hom }} e\left(u_{1}-w_{1}\right) e\left(u_{1}-w_{1}\right) \mathrm{d} x+\int_{\Omega} \int_{\Sigma_{Y}}\left(K_{\mathrm{N}}\left[u_{1}-u_{0}-w_{1}+w_{0}\right] \cdot n n\right. \\
& \left.+K_{\mathrm{T}} \sum_{i=1}^{2}\left[u_{1}-u_{0}-w_{1}+w_{0}\right] \cdot \tau^{i} \tau^{i}\right) \cdot\left(u_{1}-w_{1}-u_{0}+w_{0}\right) \mathrm{d} S(y) \mathrm{d} x \\
\geq & \alpha^{\text {hom }} C\left\|u_{1}-w_{1}\right\|_{\left[H^{1}(\Omega)\right]^{3}}^{2}+\min \left\{K_{\mathrm{N}}, K_{\mathrm{T}}\right\}\left|\Sigma_{Y}\right|\left\|u_{1}-u_{0}-w_{1}+w_{0}\right\|_{\left[L^{2}(\Omega)\right]^{3}}^{2}
\end{aligned}
$$


Thus, $u_{1}=w_{1}$. Using this and $\left\|u_{1}-u_{0}-w_{1}+w_{0}\right\|_{\left[L^{2}(\Omega)\right]^{3}}^{2}=0$, we receive $u_{0}=w_{0}$, which proves the uniqueness of the solution.

Remark 4.15. We can generalize the result using $A^{\varepsilon}=A(\cdot, \dot{\bar{\varepsilon}}) \in M(\alpha, \beta, \Omega)$ instead of $A^{\varepsilon}=$ $A(\dot{\bar{\varepsilon}}) \in M(\alpha, \beta, Y)$ and under the additional assumption that $\mathcal{T}_{1}^{\varepsilon}\left(A^{\varepsilon}\right) \rightarrow B_{1}$ a.e. in $\Omega \times Y_{1}$ and $\mathcal{T}_{0}^{\varepsilon}\left(A^{\varepsilon}\right) \rightarrow B_{0}$ a.e. in $\Omega \times Y_{0}$. Then, $B_{1} \in M\left(\alpha, \beta, \Omega \times Y_{1}\right)$ and $B_{0} \in M\left(\alpha, \beta, \Omega \times Y_{0}\right)$ and the homogenized problem is: Find the unique solutions $u_{1} \in\left[H^{1}\left(\Omega, \Gamma_{1}\right)\right]^{3}, u_{0} \in\left[L_{\text {curl }}^{2}(\Omega)\right]^{3}$ such that

$$
\begin{gathered}
\int_{\Omega} B_{1}^{\text {hom }} e\left(u_{1}\right) e\left(v_{1}\right) \mathrm{d} x+\int_{\Omega} \int_{\Sigma_{Y}}\left(K_{\mathrm{N}}\left(u_{1}-u_{0}\right) \cdot n n+K_{\mathrm{T}} \sum_{i=1}^{2}\left(u_{1}-u_{0}\right) \cdot \tau^{i} \tau^{i}\right) \cdot\left(v_{1}-v_{0}\right) \mathrm{d} S(y) \mathrm{d} x \\
=\int_{\Omega} \int_{Y_{0}} f \mathrm{~d} y \cdot v_{0} \mathrm{~d} x+\int_{\Omega} \int_{Y_{1}} f \mathrm{~d} y \cdot v_{1} \mathrm{~d} x+\int_{\Gamma_{2}} g \cdot v_{1} \mathrm{~d} S(x)
\end{gathered}
$$

with

$$
\left(B_{1}^{\mathrm{hom}}\right)_{i j k h}(x)=\int_{Y_{1}}\left(B_{1}\right)_{i j k h}(x, y)-\sum_{l, m=1}^{3}\left(B_{1}\right)_{i j l m}(x, y)\left(e_{y}\left(\chi_{1}^{k h}\right)\right)_{l m}(x, y) \mathrm{d} y
$$

and $\chi_{1}^{l m} \in\left[L^{2}\left(\Omega, H_{\mathrm{per}, 0}^{1}\left(Y_{1}\right)\right)\right]^{3}, l, m \in\{1,2,3\}$ is the unique solution of

$$
\begin{cases}\left(-\sum_{j=1}^{3} \frac{\partial}{\partial y_{j}}\left[\left(B_{1}(\cdot, y) e_{y}\left(\chi_{1}^{l m}\right)(\cdot, y)\right)_{i j}-\left(B_{1}\right)_{i j l m}(\cdot, y)\right]\right)_{1 \leq i \leq 3}=0 & \text { in } Y_{1} \\ \left(-\sum_{j=1}^{3}\left[\left(B_{1}(\cdot, y) e_{y}\left(\chi_{1}^{l m}\right)(\cdot, y)\right)_{i j}-\left(B_{1}\right)_{i j l m}(\cdot, y)\right]\right)_{1 \leq i \leq 3} \cdot n=0 & \text { on } \Sigma_{Y}\end{cases}
$$

a.e. in $\Omega$.

\subsection{Homogenization in the connected case}

In section 4.3.1, we prove a convergence result, which we apply in section 4.3 .2 to derive the homogenized problem for the connected case. In order to handle the different boundary conditions at the exterior boundary, we use a special extension operator established in [19] in the context of two-scale convergence. For some general introduction to the two-scale convergence method, we refer to [25], and we note that the two-scale convergence and weak convergence of the unfolded sequence are equivalent (cf. Theorem 4.3).

\subsubsection{Compactness result for the connected case}

The following theorem gives us some information about the two-scale limit of the solutions $u_{\alpha}^{\varepsilon}$, $\alpha \in\{0,1\}$.

Theorem 4.16. For $\alpha \in\{0,1\}$, let $\left\{u_{\alpha}^{\varepsilon}\right\}$ be a sequence in $\left[H^{1}\left(\Omega_{\alpha}^{\varepsilon}\right)\right]^{3}$ with $u_{\alpha}^{\varepsilon}=0$ on $\Gamma_{1} \cap \partial \Omega_{\alpha}^{\varepsilon}$ and

$$
\left\|e\left(u_{\alpha}^{\varepsilon}\right)\right\|_{\left[L^{2}\left(\Omega_{\alpha}^{\varepsilon}\right)\right]^{3 \times 3}} \leq C
$$

for a constant $C$ independent of $\varepsilon$. Then, there exists, up to a subsequence, $u_{\alpha} \in\left[H^{1}\left(\Omega, \Gamma_{1}\right)\right]^{3}$ and $\hat{u}_{\alpha} \in\left[L^{2}\left(\Omega, H_{\mathrm{per}}^{1}(Y) / \mathbb{R}\right)\right]^{3}$, such that

$$
\begin{aligned}
\mathcal{T}^{\varepsilon}\left(\tilde{u}_{\alpha}^{\varepsilon}\right) & \rightarrow u_{\alpha}(x) \text { weakly in }\left[L^{2}(\Omega \times Y)\right]^{3}, \\
\mathcal{T}^{\varepsilon}\left(e\left(\tilde{u}_{\alpha}^{\varepsilon}\right)\right) & \rightarrow e\left(u_{\alpha}\right)(x)+e_{y}\left(\hat{u}_{\alpha}\right)(x, y) \text { weakly in }\left[L^{2}(\Omega \times Y)\right]^{3 \times 3},
\end{aligned}
$$


where $\tilde{r}$ is the extension to $\Omega$ defined in [19, Theorem 3.5] and $\mathcal{T}^{\varepsilon}$ is the unfolding operator from [23, Definition 1.2].

Proof. Let $\left\{u_{1}^{\varepsilon}\right\}$ be a sequence in $\left[H^{1}\left(\Omega_{\alpha}^{\varepsilon}\right)\right]^{3}$ with the properties of the assumption. Using Korn's inequality ([19, Theorem 4.4]), there holds

$$
\left\|u_{\alpha}^{\varepsilon}\right\|_{\left[L^{2}\left(\Omega_{\alpha}^{\varepsilon}\right]^{3}\right.}+\left\|\nabla u_{\alpha}^{\varepsilon}\right\|_{\left[L^{2}\left(\Omega_{\alpha}^{\varepsilon}\right)\right]^{3 \times 3}} \leq C\left\|e\left(u_{\alpha}^{\varepsilon}\right)\right\|_{\left[L^{2}\left(\Omega_{\alpha}^{\varepsilon}\right]^{3 \times 3}\right.} \leq C .
$$

Therefore, $\left\{u_{\alpha}^{\varepsilon}\right\}$ is a bounded sequence in $\left[H^{1}\left(\Omega_{\alpha}^{\varepsilon}\right)\right]^{3}$ with $u_{\alpha}^{\varepsilon}=0$ in $\partial \Omega_{\alpha}^{\varepsilon} \cap \Gamma_{1}$. The extension operator defined in [19, Theorem 3.5] guarantees that $\left\{\tilde{u}_{\alpha}^{\varepsilon}\right\}$ is a bounded sequence in $\left[H^{1}\left(\Omega, \Gamma_{1}\right)\right]^{3}$. Since $\left[H^{1}\left(\Omega, \Gamma_{1}\right)\right]^{3}$ is a Hilbert space there exists a $u_{\alpha} \in\left[H^{1}\left(\Omega, \Gamma_{1}\right)\right]^{3}$ with

$$
\tilde{u}_{\alpha}^{\varepsilon} \rightarrow u_{\alpha} \text { weakly in }\left[H^{1}\left(\Omega, \Gamma_{1}\right)\right]^{3} .
$$

Therefore, we can apply $\left[25\right.$, Theorem 20] and Theorem 4.3 to get for some $\hat{u}_{\alpha} \in\left[L^{2}\left(\Omega, H_{\text {per }}^{1}(Y) / \mathbb{R}\right)\right]^{3}$

$$
\mathcal{T}^{\varepsilon}\left(\tilde{u}_{\alpha}^{\varepsilon}\right) \rightarrow u_{\alpha}(x) \text { weakly in }\left[L^{2}(\Omega \times Y)\right]^{3}
$$

and

$$
\mathcal{T}^{\varepsilon}\left(\nabla \tilde{u}_{\alpha}^{\varepsilon}\right) \rightarrow \nabla u_{\alpha}(x)+\nabla_{y} \hat{u}_{\alpha}(x, y) \text { weakly in }\left[L^{2}(\Omega \times Y)\right]^{3 \times 3} .
$$

The definition of the linear strain tensor $e\left(\tilde{u}_{\alpha}^{\varepsilon}\right)=\frac{1}{2}\left(\nabla \tilde{u}_{\alpha}^{\varepsilon}+\left(\nabla \tilde{u}_{\alpha}^{\varepsilon}\right)^{T}\right)$ directly yields

$$
\mathcal{T}^{\varepsilon}\left(e\left(\tilde{u}_{\alpha}^{\varepsilon}\right)\right) \rightarrow e\left(u_{\alpha}\right)(x)+e_{y}\left(\hat{u}_{\alpha}\right)(x, y) \text { weakly in }\left[L^{2}(\Omega \times Y)\right]^{3 \times 3} .
$$

\subsubsection{Passage to the limit in the connected case}

We endow the space

$$
\mathcal{V}\left(\Omega, Y, \Gamma_{1}\right):=\left[H^{1}\left(\Omega, \Gamma_{1}\right)\right]^{3} \times\left[L^{2}\left(\Omega, H_{\mathrm{per}, 0}^{1}\left(Y_{0}\right)\right)\right]^{3} \times\left[H^{1}\left(\Omega, \Gamma_{1}\right)\right]^{3} \times\left[L^{2}\left(\Omega, H_{\mathrm{per}, 0}^{1}\left(Y_{1}\right)\right)\right]^{3}
$$

with the norm (due to Korn's inequality for functions with zero trace on part of the boundary and for periodic functions)

$$
\|u\|_{\mathcal{V}\left(\Omega, Y, \Gamma_{1}\right)}^{2}=\left\|e\left(u_{0}\right)\right\|_{\left[L^{2}(\Omega)\right]^{3 \times 3}}^{2}+\left\|e_{y}\left(\hat{u}_{0}\right)\right\|_{\left[L^{2}\left(\Omega \times Y_{0}\right)\right]^{3 \times 3}}^{2}+\left\|u_{1}\right\|_{\left[L^{2}(\Omega)\right]^{3}}^{2}+\left\|e_{y}\left(\hat{u}_{1}\right)\right\|_{\left[L^{2}\left(\Omega \times Y_{1}\right)\right]^{3 \times 3}}^{2}
$$

for all $u=\left(u_{0}, \hat{u}_{0}, u_{1}, \hat{u}_{1}\right) \in \mathcal{V}\left(\Omega, Y, \Gamma_{1}\right)$.

Theorem 4.17. Let $\left\{u^{\varepsilon}\right\}$ be a sequence of weak solutions of the problem (3) with $u^{\varepsilon} \in \mathcal{W}_{\mathrm{c}}\left(\Omega^{\varepsilon}\right)$ and $\left\{f^{\varepsilon}\right\} \subset\left[L^{2}\left(\Omega^{\varepsilon}\right)\right]^{3}$ such that

$$
\mathcal{T}^{\varepsilon}\left(f^{\varepsilon}\right) \rightarrow f \text { weakly in }\left[L^{2}(\Omega \times Y)\right]^{3}
$$

for some $f \in\left[L^{2}(\Omega \times Y)\right]^{3}$. Let $\alpha=0$ or 1 . Then, there exist functions $u_{\alpha} \in\left[H^{1}\left(\Omega, \Gamma_{1}\right)\right]^{3}$ and $\hat{u}_{\alpha} \in\left[L^{2}\left(\Omega, H_{\text {per }}^{1}(Y) / \mathbb{R}\right)\right]^{3}$ such that

$$
\left\{\begin{array}{l}
\mathcal{T}^{\varepsilon}\left(\tilde{u}_{\alpha}^{\varepsilon}\right) \rightarrow u_{\alpha}(x) \text { weakly in }\left[L^{2}(\Omega \times Y)\right]^{3}, \\
\mathcal{T}^{\varepsilon}\left(e\left(\tilde{u}_{\alpha}^{\varepsilon}\right)\right) \rightarrow e\left(u_{\alpha}\right)(x)+e_{y}\left(\hat{u}_{\alpha}\right)(x, y) \text { weakly in }\left[L^{2}(\Omega \times Y)\right]^{3 \times 3},
\end{array}\right.
$$


where $\tilde{}$ is the extension to $\Omega$ defined in [19, Theorem 3.5] and $\mathcal{T}^{\varepsilon}$ is the unfolding operator from [23. Definition 1.2]. Furthermore, the restriction $u=\left(u_{0},\left.\hat{u}_{0}\right|_{\Omega \times Y_{0}}, u_{1},\left.\hat{u}_{1}\right|_{\Omega \times Y_{1}}\right) \in \mathcal{V}\left(\Omega, Y, \Gamma_{1}\right)$ is the solution of the problem

$$
\begin{aligned}
& \int_{\Omega} \int_{Y_{0}} A\left(e\left(u_{0}\right)+e_{y}\left(\hat{u}_{0}\right)\right)\left(e\left(v_{0}\right)+e_{y}\left(\hat{v}_{0}\right)\right) \mathrm{d} y \mathrm{~d} x+\int_{\Omega} \int_{Y_{1}} A\left(e\left(u_{1}\right)+e_{y}\left(\hat{u}_{1}\right)\right)\left(e\left(v_{1}\right)+e_{y}\left(\hat{v}_{1}\right)\right) \mathrm{d} y \mathrm{~d} x \\
& \quad+\int_{\Omega} \int_{\Sigma_{Y}}\left(K_{\mathrm{N}}\left(u_{1} \cdot n-u_{0} \cdot n\right) n+K_{\mathrm{T}} \sum_{i=1}^{2}\left(u_{1} \cdot \tau^{i}-u_{0} \cdot \tau^{i}\right) \tau^{i}\right) \cdot\left(v_{1}-v_{0}\right) \mathrm{d} S(y) \mathrm{d} x \\
& =\int_{\Omega} \int_{Y_{0}} f \mathrm{~d} y \cdot v_{0} \mathrm{~d} x+\int_{\Omega} \int_{Y_{1}} f \mathrm{~d} y \cdot v_{1} \mathrm{~d} x+\int_{\Gamma_{2}} g \cdot h_{0} v_{0} \mathrm{~d} S(x)+\int_{\Gamma_{2}} g \cdot h_{1} v_{1} \mathrm{~d} S(x)
\end{aligned}
$$

for all $v \in \mathcal{V}\left(\Omega, Y, \Gamma_{1}\right)$.

Proof. Let $\left\{u^{\varepsilon}\right\}$ be the sequence of the solutions of problem (3). We have already proven in Theorem 3.6 and Theorem 3.7 the existence, uniqueness and uniform boundedness of this sequence. So we can apply Theorem 4.16, which proves the convergences in 230 . We choose some test functions $\varphi^{\varepsilon}$ of the form

$$
\varphi_{0}^{\varepsilon}:=v_{0}+\varepsilon w_{0}^{\varepsilon} \quad \text { and } \quad \varphi_{1}^{\varepsilon}:=v_{1}+\varepsilon w_{1}^{\varepsilon}
$$

with $v_{0}, v_{1} \in\left[\mathcal{D}\left(\bar{\Omega}, \Gamma_{1}\right)\right]^{3}:=\left\{\phi \in[\mathcal{D}(\bar{\Omega})]^{3}: v\right.$ is equal to 0 in a neighbourhood of $\left.\Gamma_{1}\right\}, w_{0}^{\varepsilon}(x):=$ $w_{0}\left(x, \frac{x}{\varepsilon}\right)$ and $w_{1}^{\varepsilon}(x):=w_{1}\left(x, \frac{x}{\varepsilon}\right)$ with $w_{0}, w_{1} \in\left[\mathcal{D}\left(\Omega, C_{\text {per }}^{\infty}(Y)\right)\right]^{3}$. Therefore, $\left.\varphi_{0}^{\varepsilon}\right|_{\Gamma_{1}}=0,\left.\varphi_{1}^{\varepsilon}\right|_{\Gamma_{1}}=0$, $\varphi_{0}^{\varepsilon} \in\left[H^{1}\left(\Omega_{0}^{\varepsilon}\right)\right]^{3}$ and $\varphi_{1}^{\varepsilon} \in\left[H^{1}\left(\Omega_{1}^{\varepsilon}\right)\right]^{3}$ and the weak formulation is

$$
\begin{aligned}
\int_{\Omega_{0}^{\varepsilon}} & A^{\varepsilon} e\left(u_{0}^{\varepsilon}\right) e\left(\varphi_{0}^{\varepsilon}\right) \mathrm{d} x+\int_{\Omega_{1}^{\varepsilon}} A^{\varepsilon} e\left(u_{1}^{\varepsilon}\right) e\left(\varphi_{1}^{\varepsilon}\right) \mathrm{d} x \\
& +\varepsilon \int_{\Sigma^{\varepsilon}}\left(K_{\mathrm{N}}\left[u_{n}^{\varepsilon}\right]_{\Sigma^{\varepsilon}} n+K_{\mathrm{T}} \sum_{i=1}^{2}\left[u_{\tau^{i}}^{\varepsilon}\right]_{\Sigma^{\varepsilon}} \tau^{i}\right) \cdot\left(\varphi_{1}^{\varepsilon}-\varphi_{0}^{\varepsilon}\right) \mathrm{d} S(x) \\
\quad & =\int_{\Omega_{0}^{\varepsilon}} f^{\varepsilon} \cdot \varphi_{0}^{\varepsilon} \mathrm{d} x+\int_{\Omega_{1}^{\varepsilon}} f^{\varepsilon} \cdot \varphi_{1}^{\varepsilon} \mathrm{d} x+\int_{\Gamma_{2} \cap \partial \Omega_{0}^{\varepsilon}} g \cdot \varphi_{0}^{\varepsilon} \mathrm{d} S(x)+\int_{\Gamma_{2} \cap \partial \Omega_{1}^{\varepsilon}} g \cdot \varphi_{1}^{\varepsilon} \mathrm{d} S(x) .
\end{aligned}
$$

Let $\alpha=0$ or 1 . Using the fact that

$$
e\left(\varphi_{\alpha}^{\varepsilon}\right)(x)=e\left(v_{\alpha}\right)(x)+\varepsilon e\left(w_{\alpha}\right)\left(x, \frac{x}{\varepsilon}\right)+e_{y}\left(w_{\alpha}\right)\left(x, \frac{x}{\varepsilon}\right),
$$

and similar argumentation as in the disconnected case, we receive

$$
\mathcal{T}^{\varepsilon}\left(e\left(\varphi_{\alpha}^{\varepsilon}\right)\right) \rightarrow e\left(v_{\alpha}\right)+e_{y}\left(w_{\alpha}\right) \text { strongly in }\left[L^{2}(\Omega \times Y)\right]^{3 \times 3} .
$$

Using the periodic unfolding operator, the following convergence holds

$$
\begin{aligned}
\int_{\Omega_{\alpha}^{\varepsilon}} A^{\varepsilon} e\left(u_{\alpha}^{\varepsilon}\right) e\left(\varphi_{\alpha}^{\varepsilon}\right) \mathrm{d} x & =\int_{\Omega} \int_{Y_{\alpha}} \mathcal{T}^{\varepsilon}\left(A^{\varepsilon}\right) \mathcal{T}^{\varepsilon}\left(e\left(\tilde{u}_{\alpha}^{\varepsilon}\right)\right) \mathcal{T}^{\varepsilon}\left(e\left(\varphi_{\alpha}^{\varepsilon}\right)\right) \mathrm{d} x \\
& \longrightarrow \int_{\Omega} \int_{Y_{\alpha}} A\left(e\left(u_{\alpha}\right)+e_{y}\left(\hat{u}_{\alpha}\right)\right)\left(e\left(v_{\alpha}\right)+e_{y}\left(w_{\alpha}\right)\right) \mathrm{d} y \mathrm{~d} x .
\end{aligned}
$$


We receive the convergence of the interface term from Lemma 4.18 and the fact that the terms with $w_{1}^{\varepsilon}$ and $w_{0}^{\varepsilon}$ vanish. Moreover,

$$
\int_{\Omega_{\alpha}^{\varepsilon}} f^{\varepsilon} \cdot \varphi_{\alpha}^{\varepsilon} \mathrm{d} x=\int_{\Omega} \int_{Y_{\alpha}} \mathcal{T}^{\varepsilon}\left(f^{\varepsilon}\right) \cdot \mathcal{T}^{\varepsilon}\left(v_{\alpha}\right) \mathrm{d} x+\varepsilon \int_{\Omega} \int_{Y_{\alpha}} \mathcal{T}^{\varepsilon}\left(f^{\varepsilon}\right) \cdot \mathcal{T}^{\varepsilon}\left(w_{\alpha}^{\varepsilon}\right) \mathrm{d} x \longrightarrow \int_{\Omega} \int_{Y_{\alpha}} f \mathrm{~d} y \cdot v_{\alpha} \mathrm{d} x .
$$

For the proof of the convergence of the boundary integral we refer to section 7.6.2 in [19]

$$
\int_{\Gamma_{2} \cap \partial \Omega_{\alpha}^{\varepsilon}} g \cdot \varphi_{\alpha}^{\varepsilon} \mathrm{d} S(x) \longrightarrow \int_{\Gamma_{2}} g \cdot h_{\alpha} v_{\alpha} \mathrm{d} S(x)
$$

for some $h_{\alpha} \in L^{2}\left(\Gamma_{2}\right)$. Under further assumption on the boundary $\partial \Omega$ and the exterior boundaries of $Y_{0}$ and $Y_{1}$ we can formulate the limit functions $h_{0}$ and $h_{1}$ explicitly (see [19, Theorem 7.17]). Using the convergence results above and the density of $\left[\mathcal{D}\left(\bar{\Omega}, \Gamma_{1}\right)\right]^{3}$ in $\left[H^{1}\left(\Omega, \Gamma_{1}\right)\right]^{3}$ (see $[26$, Theorem 3.1]) and $\left[\mathcal{D}\left(\Omega, C_{\text {per }}^{\infty}(Y)\right)\right]^{3}$ in $\left[L^{2}\left(\Omega, H_{\text {per }}^{1}(Y)\right)\right]^{3}$, we get the desired homogenized problem 24 up to uniqueness. To ensure the uniqueness, we can restrict the space $\left[L^{2}\left(\Omega, H_{\text {per }}^{1}(Y) / \mathbb{R}\right)\right]^{3}$ to $\left[L^{2}\left(\Omega, H_{\text {per }, 0}^{1}\left(Y_{0}\right)\right)\right]^{3}$ resp. $\left[L^{2}\left(\Omega, H_{\text {per }, 0}^{1}\left(Y_{1}\right)\right)\right]^{3}$ for the functions $\hat{u}_{0}, \hat{u}_{1}$ and the test functions in the limit problem, which does not change the limit problem. Since the solution is unique, which we prove below, all the convergences above hold true for the whole sequence.

We want to prove the limit result for the interface term used in the proof of the last theorem.

Lemma 4.18. Let $\left\{u^{\varepsilon}\right\}$ be the sequence as in Theorem 4.17. Then, there holds up to a subsequence for all $v_{0}, v_{1} \in\left[\mathcal{D}\left(\bar{\Omega}, \Gamma_{1}\right)\right]^{3}$

$$
\begin{aligned}
& \varepsilon \int_{\Sigma^{\varepsilon}}\left(K_{\mathrm{N}}\left[\tilde{u}_{1}^{\varepsilon} \cdot n-\tilde{u}_{0}^{\varepsilon} \cdot n\right] n+K_{\mathrm{T}} \sum_{i=1}^{2}\left[\tilde{u}_{1}^{\varepsilon} \cdot \tau^{i}-\tilde{u}_{0}^{\varepsilon} \cdot \tau^{i}\right] \tau^{i}\right) \cdot\left(v_{1}-v_{0}\right) \mathrm{d} S(x) \\
& \stackrel{\varepsilon \rightarrow 0}{\longrightarrow} \int_{\Omega} \int_{\Sigma_{Y}}\left(K_{\mathrm{N}}\left[u_{1} \cdot n-u_{0} \cdot n\right] n+K_{\mathrm{T}} \sum_{i=1}^{2}\left[u_{1} \cdot \tau^{i}-u_{0} \cdot \tau^{i}\right] \tau^{i}\right) \cdot\left(v_{1}-v_{0}\right) \mathrm{d} S(y) \mathrm{d} x,
\end{aligned}
$$

whereby only $n$ and $\tau^{i}, i=1,2$, depend on $y$.

Proof. If we apply the boundary unfolding operator $\mathcal{T}_{\mathrm{b}}^{\varepsilon}$ defined in [23, Definition 3.1] we get

$$
\varepsilon K_{\mathrm{N}} \int_{\Sigma^{\varepsilon}} \tilde{u}_{1}^{\varepsilon} \cdot n v_{1} \cdot n \mathrm{~d} S(x)=\sum_{i, j=1}^{3} \frac{K_{\mathrm{N}}}{|Y|} \int_{\Omega \times \Sigma_{Y}} \mathcal{T}^{\varepsilon}\left(\tilde{u}_{1}^{\varepsilon}\right)_{i} \mathcal{T}_{\mathrm{b}}^{\varepsilon}\left(n_{i}\right) \mathcal{T}_{\mathrm{b}}^{\varepsilon}\left(n_{j}\right) \mathcal{T}^{\varepsilon}\left(v_{1}\right)_{j} \mathrm{~d} S(y) \mathrm{d} x .
$$

Using the fact that $\mathcal{T}_{\mathrm{b}}^{\varepsilon}(n)(x, y)=n(y)$ for all $(x, y) \in \Omega \times \Sigma_{Y}$ and Proposition 4.2 applied to $v_{1}$, we can conclude that

$$
\varepsilon K_{\mathrm{N}} \int_{\Sigma^{\varepsilon}} \tilde{u}_{1}^{\varepsilon} \cdot n v_{1} \cdot n \mathrm{~d} S(x) \longrightarrow K_{\mathrm{N}} \int_{\Omega} \int_{\Sigma_{Y}} u_{1} \cdot n n \cdot v_{1} \mathrm{~d} S(y) \mathrm{d} x .
$$

Following the same steps of the proof for all other summands of (25), we get the desired result.

We want to split the problem (24) into a micro- and macroscopic problem. 
Theorem 4.19. Let $\left\{u^{\varepsilon}\right\}$ be as in Theorem 4.17. We can reformulate the homogenized problem 24): Find $u_{0}, u_{1} \in\left[H^{1}\left(\Omega, \Gamma_{1}\right)\right]^{3}$ with

$$
\begin{aligned}
& \int_{\Omega} A_{0}^{\text {hom }} e\left(u_{0}\right) e\left(v_{0}\right) \mathrm{d} x+\int_{\Omega} A_{1}^{\text {hom }} e\left(u_{1}\right) e\left(v_{1}\right) \mathrm{d} x \\
& \quad+\int_{\Omega} \int_{\Sigma_{Y}}\left(K_{\mathrm{N}}\left(u_{1} \cdot n-u_{0} \cdot n\right) n+K_{\mathrm{T}} \sum_{i=1}^{2}\left(u_{1} \cdot \tau^{i}-u_{0} \cdot \tau^{i}\right) \tau^{i}\right) \cdot\left(v_{1}-v_{0}\right) \mathrm{d} S(y) \mathrm{d} x \\
& \quad=\int_{\Omega} \int_{Y_{0}} f \mathrm{~d} y \cdot v_{0} \mathrm{~d} x+\int_{\Omega} \int_{Y_{1}} f \mathrm{~d} y \cdot v_{1} \mathrm{~d} x+\int_{\Gamma_{2}} g \cdot h_{0} v_{0} \mathrm{~d} S(x)+\int_{\Gamma_{2}} g \cdot h_{1} v_{1} \mathrm{~d} S(x)
\end{aligned}
$$

whereby

$$
\left(A_{\alpha}^{\mathrm{hom}}\right)_{i j k h}=\int_{Y_{\alpha}} a_{i j k h}(y)-\sum_{l, m=1}^{3} a_{i j l m}\left(e_{y}\left(\chi_{\alpha}^{k h}\right)\right)_{l m} \mathrm{~d} y
$$

and $\chi_{\alpha}^{l m} \in\left[H^{1}\left(Y_{\alpha}\right)\right]^{3}, l, m \in\{1,2,3\}$ is the unique solution of

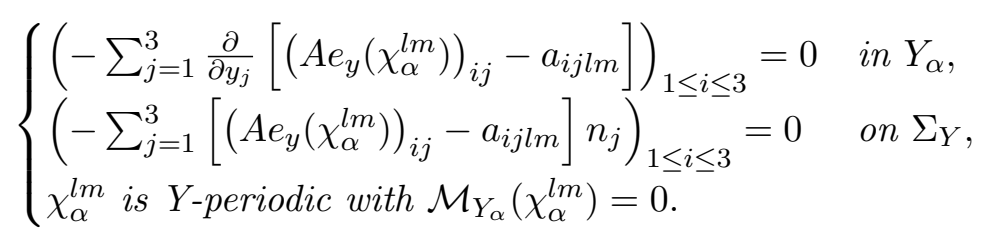

for $\alpha \in\{0,1\}$.

Proof. This result can be shown in the same way as in the proof of Theorem 4.13.

Similar to [17, Theorem II.1.1], it can be proven that there exist $\alpha_{0}^{\text {hom }}, \beta_{0}^{\text {hom }}, \alpha_{1}^{\text {hom }}, \beta_{1}^{\text {hom }} \in$ $\mathbb{R}$ with $0<\alpha_{0}^{\text {hom }}<\beta_{0}^{\text {hom }}, 0<\alpha_{1}^{\text {hom }}<\beta_{1}^{\text {hom }}$ such that $A_{0}^{\text {hom }} \in M\left(\alpha_{0}^{\text {hom }}, \beta_{0}^{\text {hom }}, \Omega\right), A_{1}^{\text {hom }} \in$ $M\left(\alpha_{1}^{\text {hom }}, \beta_{1}^{\text {hom }}, \Omega\right)$. With this fact we are able to prove the uniqueness of the solution of 26 .

Theorem 4.20. There exist unique solutions $u_{0}, u_{1} \in\left[H^{1}\left(\Omega, \Gamma_{1}\right)\right]^{3}$ of the macroscopic problem (26).

Proof. The proof is analogous to that of Theorem 4.14 .

Remark 4.21. As in the disconnected case, we can generalize the result under the assumptions that $A^{\varepsilon}=A(\cdot, \dot{\bar{\varepsilon}}) \in M(\alpha, \beta, \Omega)$ and $\mathcal{T}_{1}^{\varepsilon}\left(A^{\varepsilon}\right) \rightarrow B_{1}$ a.e. in $\Omega \times Y_{1}$ and $\mathcal{T}_{0}^{\varepsilon}\left(A^{\varepsilon}\right) \rightarrow B_{0}$ a.e. in $\Omega \times Y_{0}$. The homogenized problem is the same as above but the cell problems and the definition of the homogenized tensors $A_{0}^{\text {hom }}$ and $A_{1}^{\text {hom }}$ need to be adjusted in an analogous fashion as in Remark 4.15.

\section{Comparison of the homogenized problem in the disconnected and connected case and discussion}

In this section, we state and compare the strong formulations of the upscaled problems in the disconnected and connected case and discuss their differences.

In the disconnected case, we only know so far that the solution $u_{0}$ is unique if $\nabla \times u_{0}=0$ (see Theorem 4.14). Thus, we have to prove that a general solution already satisfies the curlfree condition, which is physically expected owing to the coupling of $u_{0}$ and $u_{1}$ through the slip 
displacement conditions. (The disconnected components cannot undergo rigid-body motions due to the bond to the connected part.) By using the unique decomposition of functions $v \in L^{2}(\Omega)$, namely $v=\nabla p+\nabla \times w$ for some functions $p, w$ (for more details we refer to [27]), the interface term yields the desired result. In the disconnected case (cf. eq. (21)), the strong formulation is given by: Find $u_{1}, u_{0}$ such that

$$
\begin{aligned}
-\nabla \cdot\left(A_{1}^{\mathrm{hom}} e\left(u_{1}\right)\right)+\int_{\Sigma_{Y}} K_{\mathrm{N}}\left(u_{1}-u_{0}\right) \cdot n n+K_{\mathrm{T}} \sum_{i=1}^{2}\left(u_{1}-u_{0}\right) \cdot \tau^{i} \tau^{i} \mathrm{~d} S(y) & =\int_{Y_{1}} f \mathrm{~d} y & & \text { in } \Omega, \\
-\int_{\Sigma_{Y}} K_{\mathrm{N}}\left(u_{1}-u_{0}\right) \cdot n n+K_{\mathrm{T}} \sum_{i=1}^{2}\left(u_{1}-u_{0}\right) \cdot \tau^{i} \tau^{i} \mathrm{~d} S(y) & =\int_{Y_{0}} f \mathrm{~d} y & & \text { in } \Omega, \\
A_{1}^{\mathrm{hom}} e\left(u_{1}\right) \cdot n & =g & & \text { on } \Gamma_{2}, \\
u_{1} & =0 & & \text { on } \Gamma_{1},
\end{aligned}
$$

while in the connected case (cf. eq. (26)), it is: Find $u_{0}, u_{1}$ such that

$$
\begin{array}{rlrl}
-\nabla \cdot\left(A_{1}^{\mathrm{hom}} e\left(u_{1}\right)\right)+\int_{\Sigma_{Y}} K_{\mathrm{N}}\left(u_{1}-u_{0}\right) \cdot n n+K_{\mathrm{T}} \sum_{i=1}^{2}\left(u_{1}-u_{0}\right) \cdot \tau^{i} \tau^{i} \mathrm{~d} S(y) & =\int_{Y_{1}} f \mathrm{~d} y & \text { in } \Omega, \\
-\nabla \cdot\left(A_{0}^{\mathrm{hom}} e\left(u_{0}\right)\right)-\int_{\Sigma_{Y}} K_{\mathrm{N}}\left(u_{1}-u_{0}\right) \cdot n n+K_{\mathrm{T}} \sum_{i=1}^{2}\left(u_{1}-u_{0}\right) \cdot \tau^{i} \tau^{i} \mathrm{~d} S(y) & =\int_{Y_{0}} f \mathrm{~d} y & & \text { in } \Omega, \\
A_{0}^{\mathrm{hom}} e\left(u_{0}\right) \cdot n & =g h_{0} & & \text { on } \Gamma_{2}, \\
A_{1}^{\mathrm{hom}} e\left(u_{1}\right) \cdot n & =g h_{1} & & \text { on } \Gamma_{2}, \\
u_{1} & =0 & & \text { on } \Gamma_{1}, \\
u_{0} & =0 & & \text { on } \Gamma_{1} .
\end{array}
$$

Apart from the different boundary conditions at the exterior boundary, the main difference is that, in the disconnected case, there is no contribution of any homogenized tensor $A_{0}^{\text {hom }}$. This means that no stress is transmitted globally by the material occupying the disconnected domain. Moreover, adding the two equations stated in $\Omega$ for the disconnected case makes the interfacial terms disappear and leaves only the total volumetric forcing given by $f$ in the homogenized elasticity equation for $u_{1}$. Nevertheless, as $f$ is a force density w.r.t. volume, the density of the material does play a role. Similarly, inertial effects are expected to have an impact in the associated timedependent case.

In terms of the application to concretes reinforced with short carbon fibres, it is unlikely that the disconnected case is the correct model. However, it may be anticipated that modelling the carbon fibres as connected in only one spatial dimension but not in the other two might lead to a mixture of the two cases, which may lead to appropriate models if realistic unit cells are used. This, together with simulation results comparing to experimental data, is planned as future work.

\section{Acknowledgements}

This work was supported by the German Research Foundation (DFG) through a project within the Special Priority Program (SPP) 2020. 


\section{References}

[1] M. Hambach, H. Möller, T. Neumann, D. Volkmer, Portland cement paste with aligned carbon fibers exhibiting exceptionally high flexural strength (> $100 \mathrm{MPa}$ ), Cement and Concrete Research 89 (2016) 80-86.

[2] P. Lauff, M. Raith, C. Grosse, M. Rutzen, D. Volkmer, L. Reischmann, U. Weiß, M. A. Peter, O. Fischer, Investigation of localized damage indicators of a short-fibre reinforced high performance concrete under dynamic and flexural load, in: W. Derkowski et al. (Ed.), Concrete: Innovations in Materials, Design and Structures Proceedings of the fib Symposium 2019, Kraków, Poland, 27-29 May 2019, pp. 1952-1959.

[3] M. Rutzen, D. Volkmer, U. Weiß, L. Reischmann, M. A. Peter, P. Lauff, O. Fischer, M. Raith, C. Große, Microstructural analysis of crack growth caused by static and dynamic loads in a carbon fiber reinforced cement past, in: A. Zingoni (Ed.), Advances in Engineering Materials, Structures and Systems: Innovations, Mechanics and Applications, 2019, pp. 617-622.

[4] P. Donato, S. Monsurrò, Homogenization of two heat conductors with an interfacial contact barrier, Analysis and Applications 02 (03) (2004) 247-273.

[5] P. Donato, K. H. Le Nguyen, R. Tardieu, The periodic unfolding method for a class of imperfect transmission problems., J. Math. Sci., New York 176 (6) (2011) 891-927.

[6] R. Bunoiu, C. Timofte, Upscaling of a diffusion problem with interfacial flux jump leading to a modified barenblatt model, ZAMM - Journal of Applied Mathematics and Mechanics / Zeitschrift für Angewandte Mathematik und Mechanik 99 (2) (2018).

[7] L. Tartar, Quelques remarques sur l'homogénéisation, functional analysis and numerical analysis, Proc. JapanFrance Seminar 1976 (1978) 468-482.

[8] D. Cioranescu, A. Damlamian, G. Griso, The periodic unfolding method in homogenization., SIAM J. Math. Anal. 40 (4) (2008) 1585-1620.

[9] M. A. Peter, M. Böhm, Different choices of scaling in homogenization of diffusion and interfacial exchange in a porous medium, Mathematical Methods in the Applieds Sciences 31 (2008) 1257-1282.

[10] G. Nguetseng, A general convergence result for a functional related to the theory of homogenization, SIAM Journal on Mathematical Analysis 20 (3) (1989) 608-629.

[11] G. Allaire, Homogenization and two-scale convergence., SIAM J. Math. Anal. 23 (6) (1992) 1482-1518.

[12] P. Donato, K. H. Le Nguyen, Homogenization of diffusion problems with a nonlinear interfacial resistance, Nonlinear Differ. Equ. Appl. 22 (2015) 1345-1380.

[13] K. H. Le Nguyen, Homogenization of of heat transfer process in composite materials, J. Elliptic Parabolic Equations 1 (2015) 175-188.

[14] I. Graf, M. A. Peter, J. Sneyd, Homogenization of a nonlinear multiscale model of calcium dynamics in biological cells, Journal of Mathematical Analysis and Applications 419 (1) (2014) 28-47.

[15] J. Orlik, Two-scale homogenization in transmission problems of elasticity with interface jumps, Applicable Analysis 91 (7) (2011) 1299-1319.

[16] M. Ptashnyk, B. Seguin, Homogenization of a system of elastic and reaction-diffusion equations modelling plant cell wall biomechanics, ESAIM: Mathematical Modelling and Numerical Analysis 50 (2) (2016) 593-631.

[17] O. Oleinik, A. Shamaev, G. Yosifian, Mathematical problems in elasticity and homogenization, North-Holland, Amsterdam, London, New York, Tokyo, 1992.

[18] D. Cioranescu, P. Donato, An Introduction to Homogenization, Oxford Univ. Press, Oxford [u.a], 1999.

[19] M. Höpker, Extension operators for sobolev spaces on periodic domains, their applications, and homogenization of a phase field model for phase transitions in porous media, PhD thesis, University of Bremen (2016).

[20] B. Lombard, J. Piraux, Numerical modeling of elastic waves across imperfect contacts., SIAM J. Sci. Comput. 28 (1) (2006) 172-205.

[21] G. Alessandrini, A. Morassi, E. Rosset, The linear constraints in Poincaré and Korn type inequalities., Forum Math. 20 (3) (2008) 557-569.

[22] S. Monsurrò, Homogenization of a two-component composite with interfacial thermal barrier, Adv. in Math. Sci. and Appl. 13 (01) (2003) 43-63.

[23] D. Cioranescu, A. Damlamian, P. Donato, G. Griso, R. Zaki, The periodic unfolding method in domains with holes., SIAM J. Math. Anal. 44 (2) (2012) 718-760.

[24] D. Cioranescu, A. Damlamian, G. Griso, The periodic unfolding method, Springer, Singapore, 2018.

[25] D. Lukkassen, G. Nguetseng, P. Wall, Two-scale convergence, Int. J. of Pure and Appl. Math.. 2 (1) (2002) $35-86$.

[26] J.-M. E. Bernard, Density results in Sobolev spaces whose elements vanish on a part of the boundary., Chin. Ann. Math., Ser. B 32 (6) (2011) 823-846. 
[27] R. Dautray, J.-L. Lions, Mathematical Analysis and Numerical Methods for Science and Technology, Springer, Berlin, Heidelberg, 2000. 